ARTICLE

Received 8 Dec 2016 | Accepted 21 Feb 2017 | Published 28 Apr 2017

DOI: $10.1038 /$ ncomms15008 OPEN

\title{
HMGA1 amplifies Wnt signalling and expands the intestinal stem cell compartment and Paneth cell niche
}

Lingling Xian¹, Dan Georgess², Tait Huso', Leslie Cope ${ }^{3}$, Amy Belton¹, Yu-Ting Chang ${ }^{4}$, Wenyong Kuang ${ }^{1}$, Qihua Gu1, Xiaoyan Zhang ${ }^{1}$, Stefania Senger ${ }^{5}$, Alessio Fasano ${ }^{5}$, David L. Huso ${ }^{6, \ddagger}$, Andrew J. Ewald ${ }^{2,7}$

\& Linda M.S. Resar ${ }^{1,7,8}$

High-mobility group A1 (Hmga1) chromatin remodelling proteins are enriched in intestinal stem cells (ISCs), although their function in this setting was unknown. Prior studies showed that Hmga1 drives hyperproliferation, aberrant crypt formation and polyposis in transgenic mice. Here we demonstrate that Hmga1 amplifies $\mathrm{Wnt} / \beta$-catenin signalling to enhance selfrenewal and expand the ISC compartment. Hmga1 upregulates genes encoding both Wnt agonist receptors and downstream Wnt effectors. Hmgal also helps to 'build' an ISC niche by expanding the Paneth cell compartment and directly inducing Sox9, which is required for Paneth cell differentiation. In human intestine, HMGA1 and SOX9 are positively correlated, and both become upregulated in colorectal cancer. Our results define a unique role for Hmga1 in intestinal homeostasis by maintaining the stem cell pool and fostering terminal differentiation to establish an epithelial stem cell niche. This work also suggests that deregulated Hmgar perturbs this equilibrium during intestinal carcinogenesis.

\footnotetext{
${ }^{1}$ Division of Hematology, Department of Medicine, The Johns Hopkins University School of Medicine, 720 Rutland Avenue, Ross Research Building, Room 1025, Baltimore, Maryland 21205, USA. ${ }^{2}$ Department of Cell Biology, The Johns Hopkins University School of Medicine, 855 North Wolfe Street, Baltimore, Maryland 21205, USA. ${ }^{3}$ Division of Biostatistics, Department of Oncology, The Johns Hopkins University School of Medicine, 550 North Broadway, Baltimore, Maryland 21205, USA. ${ }^{4}$ Department of Pathology, Pathobiology Graduate Program, The Johns Hopkins University School of Medicine, 720 Rutland Avenue, Ross Research Building, Room 1025, Baltimore, Maryland 21205, USA. ${ }^{5}$ Department of Pediatrics, Mucosal Immunology and Biology Research Center, Harvard Medical School, Massachusetts General Hospital East, 16th Street, Building 114, Charlestown, Massachusetts 02114, USA. ${ }^{6}$ Department of Molecular and Comparative Pathobiology, The Johns Hopkins University School of Medicine, Baltimore, Maryland 21205, USA. ${ }^{7}$ Department of Oncology, The Johns Hopkins University School of Medicine, Baltimore, Maryland 21205, USA. ${ }^{8}$ Department of Pathology and Institute for Cellular Engineering, The Johns Hopkins University School of Medicine, Baltimore, Maryland 21205, USA. Correspondence and requests for materials should be addressed to L.M.S.R. (email: Iresar@jhmi.edu).

$\ddagger$ Deceased.
} 
ntestinal stem cells (ISCs) provide a paradigm for studying adult stem cell function due to their exceptional self-renewal potential and repetitive structural organization ${ }^{1-5}$. Indeed, the intestinal lining is among the most highly regenerative tissues, renewing itself every 3-5 days to protect the gut from pathogens and maintain nutrient intake essential for life. Over the past decade, a population of self-renewing, columnar epithelial cells located at the base of the intestinal crypts has been identified and characterized as ISCs ${ }^{1-5}$. They are marked by the serpentine receptor, leucine-rich repeat containing G-protein-coupled receptor 5 (Lgr5), which mediates Wnt signalling cues from the niche ${ }^{5}$. Lineage tracing experiments demonstrate that these ISCs are responsible for the exuberant regeneration and tissue homeostasis in intestinal epithelium ${ }^{1,4,6}$. Despite extensive study, the molecular mechanisms that govern their behaviour are only beginning to be elucidated ${ }^{1-9}$. Previous work also demonstrates that aberrant expression or mutation of key regulators of ISCs leads to neoplastic growth and intestinal carcinogenesis $^{10,11}$.

Emerging evidence highlights the central role for chromatin structure and chromatin-binding proteins in maintaining stem cell properties. In fact, recent work found that the high-mobility group A1 chromatin remodelling proteins (HMGA1, formerly HMG-I/Y) regulate stem cell properties in cancer ${ }^{12-18}$, although their role in normal development has remained elusive. The HMGA1 gene encodes the HMGA1a and HMGA1b isoforms ${ }^{19-21}$, which function as architectural transcription factors. HMGA1 proteins bind to specific DNA sequences ${ }^{13,22-24}$, modulate chromatin structure ${ }^{23}$ and recruit other transcriptional complexes to regulatory regions throughout the genome $e^{13,22,23}$. HMGA1 is highly expressed during embryogenesis, with high levels in normal embryonic stem cells ${ }^{13,16,25,26}$. Postnatally, HMGA1 is expressed in adult stem cells, such as hematopoietic $^{27,28}$ and intestinal stem cells ${ }^{29}$, but absent or barely detectable in mature, differentiated tissues. In cancer, HMGA1 becomes aberrantly expressed through oncogenic transcription factors and epigenetic alterations, or in rare cases, chromosomal translocation events ${ }^{13,17,30,31}$. Moreover, HMGA1 is overexpressed in most high-grade or poorly differentiated cancers studied to date, and high levels portend a poor prognosis in diverse tumours ${ }^{12-18,26,31-36}$. In murine tumour xenografts, HMGA1 drives tumour progression and cancer stem cell properties, at least in part, by inducing stem cell transcriptional networks ${ }^{12-18}$. In human embryonic stem cells, HMGA1 maintains a de-differentiated state by upregulating genes involved in stemness and pluripotency ${ }^{16}$. Moreover, HMGA1 is required for reprogramming somatic cells to induced pluripotent stem cells by the Yamanaka factors; disrupting HMGA1 expression or function prevents the derivation of fully reprogrammed cells ${ }^{16}$. Given its dual role in normal development and cancer, further studies to dissect HMGA1 function in each setting are needed to determine the therapeutic potential of targeting HMGA1 in cancer or harnessing its function for tissue regeneration.

We previously demonstrated that transgenic mice overexpressing murine $\mathrm{Hmgal}$ from the $\mathrm{H}-2 \mathrm{~K}^{\mathrm{b}}$ promoter and immunoglobulin $\mu$ enhancer all succumb to lymphoid tumours ${ }^{35}$; females also develop uterine sarcomas ${ }^{36}$. In this model, the transgene is expressed in the intestines ${ }^{14}$ in addition to lymphoid cells ${ }^{35}$ and uterine tissue ${ }^{36}$. The Hmgal transgenics develop marked proliferative changes in the epithelium of the small and large intestine, with aberrant crypt formation and polyposis ${ }^{14}$. To determine how Hmgal disrupts tissue homeostasis in the intestines of transgenic mice and intestinal cancers overexpressing HMGA1, we examined its expression and function in our transgenic model and in intestinal organoids.
We discover that Hmgal expands the ISC pool and Paneth cell niche in vivo. Hmgal is a key factor involved in the organization of ISCs into three-dimensional (3D) organoids in vitro. We also find that Hmgal enhances ISC expansion and self-renewal by amplifying Wnt/ $\beta$-catenin signalling. Hmgal also directly upregulates Sox9 and expands the Paneth cell niche. This is an example of Hmgal fostering terminal differentiation to establish a stem cell niche. Moreover, both HMGA1 and SOX9 are positively correlated in human intestinal epithelium, and both become markedly upregulated in colorectal cancer. These results reveal a unique role for Hmgal in maintaining both the ISC pool and niche cells within intestinal crypts and suggest that this equilibrium is perturbed when Hmgal becomes deregulated during carcinogenesis.

\section{Results}

Hmgal drives expansion of the ISC compartment. A prior gene expression profile study showed that Hmgal is among the genes enriched in Lgr5 + ISCs (ref. 29). HMGA1 is also among the genes most highly expressed in diverse epithelial human cancers as compared to normal epithelium, including intestinal malignancies $^{12-14,17,33}$. We therefore sought to elucidate the functional role of Hmgal in ISCs, both in normal intestinal epithelial homeostasis and in intestinal neoplasia. To this end, we crossed our Hmgal transgenic mice onto Lgr5-EGFP mice ${ }^{6}$, which mark Lgr5 + ISCs with enhanced green fluorescent protein (EGFP). The Hmgal transgene is driven by the $\mathrm{H}-2 \mathrm{~K}^{\mathrm{b}}$ promoter and $\mu$ enhancer, which confer transgene expression in intestinal crypt basilar cells ${ }^{37}$, lymphoid cells ${ }^{35}$ and uterine tissue ${ }^{36}$. In both Hmgal transgenic and wild-type (WT) mice, Hmgal protein localizes to the nuclei of Lgr5 + ISCs (Fig. 1a-d). Interestingly, Lgr5 + ISCs extend further up the crypts in the Hmgal transgenic mice compared to WT mice in all regions of the small intestine (duodenum, jejunum, ileum; Fig. la-f), consistent with the expansion in the ISC pool and enhanced self-renewal in vivo. The percentage of Lgr5 + ISCs per total crypt cells by fluorescent stain (Fig. 1f; ${ }^{* *} P<0.00001$; Mann-Whitney test) and relative frequency of Lgr5 + ISCs per crypt cell isolates by fluorescenceactivated cell sorting (FACS) were significantly $(P<0.05$; two-tailed Student's $t$-test) increased in the Hmgal transgenic small intestine (Fig. 1g,h), further validating the expansion in ISCs. To determine whether Hmgal gene expression accounts for the elevated protein levels in the Lgr5 + ISCs, we assessed Hmgal mRNA in WT mice and found that it is increased by $\sim 4$-fold in Lgr5 + ISCs isolated by FACS compared to Lgr5 - cells (Fig. 1g,h). We also assessed Hmgal mRNA in crypt cells from the transgenic model and found that Hmgal expression is increased about twofold in both Lgr5+ ISCs and Lgr5 - cells compared to the same cell populations in WT mice. In both WT and transgenic models, Hmgal mRNA is enriched in the Lgr5 + ISC population (Fig. 1g,h).

ISCs are regulated by factors from the stromal compartment in addition to intestinal epithelial cells ${ }^{1-9,38-40}$. To define the role of Hmgal in ISCs within the epithelial compartment, we used organoids, an in vitro intestinal crypt cell culture model ${ }^{1-4,41}$. Organoid buds are a surrogate for ISC function because they comprise crypt-like structures with ISCs on the tips; differentiated epithelial cells extend towards the luminal centres of the organoids. We derived organoids from small intestinal epithelial crypt cells isolated from transgenic or WT mice and compared bud formation and projected surface area. Prior to culture, an equal number of crypts from WT or Hmgal transgenic mice of similar sizes were isolated by passage through a $70 \mu \mathrm{M}$ filter ${ }^{41}$. Similar to the histologic results in the transgenic mouse intestine, we found a marked increase in projected surface area per organoid (Image-Pro Plus Version 6) and bud number per 

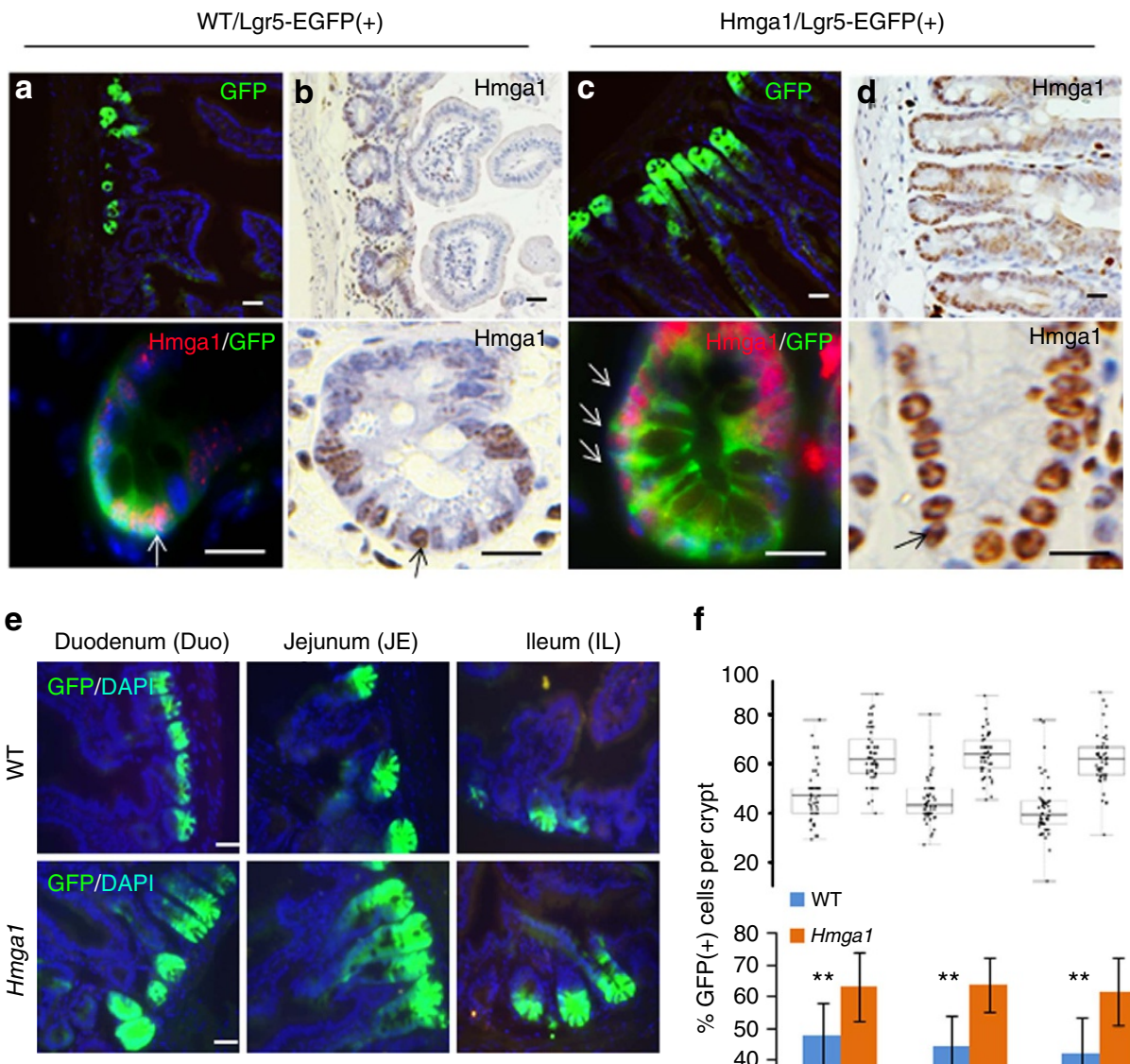

f

e

g
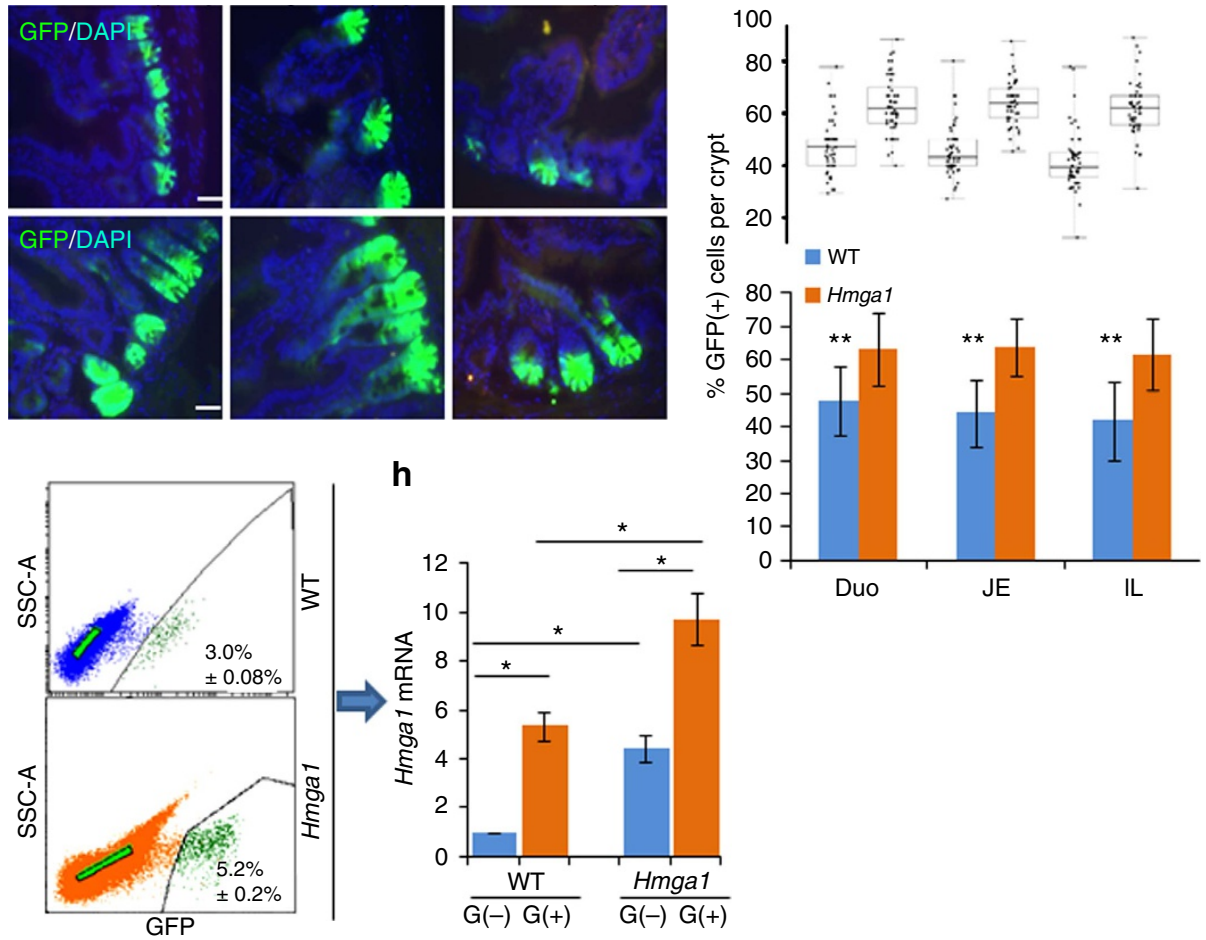

Figure 1 | Hmga1 localizes to ISCs and its overexpression expands the ISC compartment in transgenic mice. (a) ISCs from WT/Lgr5-EGFP + mice stain green (upper panel) in small intestinal cross-sections. Hmga1 stains red and localizes to GFP + ISCs (lower panel). (b) Intranuclear Hmga1 stains brown (immunohistochemistry (IHC)) in small intestinal cross-sections from WT/Lgr5-EGFP + mice. (c) ISCs stain green in Hmga1/Lgr5-EGFP + transgenic mice and extend further up the base of the crypt in Hmgal transgenic mice compared to WT mice. (Cells with red nuclear staining outside of crypts are Hmga1 transgenic lymphocytes) (d) Intranuclear Hmga1 stains brown (IHC) in small intestinal cross-sections from Hmga1/Lgr5-EGFP + mice.

(e) Fluorescent staining identifies GFP + ISCs at each region in the small intestine of WT and Hmga1 mice (duodenum, jejunum, ileum). (f) The percentage (mean \pm s.d.) of GFP + ISCs per crypt in WT and Hmgal transgenic mice are shown; ${ }^{\star \star} P<0.00001$, Mann-Whitney test $(n=50$ crypts per region, 3 mice per genotype). Dot plot shows individual data points. (g) Hmgal transgenic mice have higher GFP + ISC frequency as assessed by flow cytometry; mean frequency \pm s.d. from two experiments are shown. (h) Hmga1 mRNA is enriched in ISCs isolated by flow cytometry for GFP + cells using quantitative, real-time PCR (qPCR) in WT and Hmgal transgenic mice. Hmgal is higher in both GFP + ISCs and GFP - crypt cells from the Hmgal transgenic model compared to WT mice. Bars show mean relative Hmgal expression \pm s.d. from three experiments performed in triplicate; Gapdh was used as a loading control; * $P<0.05$; two-tailed Student's $t$-test. Scale bars, $20 \mu \mathrm{m}$.

organoid in those derived from the Hmgal transgenic mice as compared to WT controls (Fig. 2a-c; Supplementary Fig. 1a,b). There were more Hmgal organoids with $>3$ buds as compared to WT organoids by day 6 in culture (Fig. 2b). The majority of WT organoids had $\leq 1$ buds per organoid, while the majority of Hmgal organoids had $\geq 2$ buds at day 6 (Fig. 2b). Next, we isolated small crypts by passage through a $40 \mu \mathrm{M}$ filter ${ }^{40,41}$ and performed time-lapsed confocal microscopy ${ }^{42-45}$ to ascertain the rate of self-renewal in Lgr5 + ISCs and bud development from day 0 to day 6 in $3 \mathrm{D}$ organoid culture (Fig. $2 \mathrm{~d}-\mathrm{f}$ ). A representative image of crypt cells from days 0 and 6 from WT mice or Hmgal transgenics, respectively, are shown (Fig. 2d). We discovered a marked increase in the rate of self-renewal at day 6 in the Lgr5 + ISCs from the Hmgal transgenics compared to the WT 
a

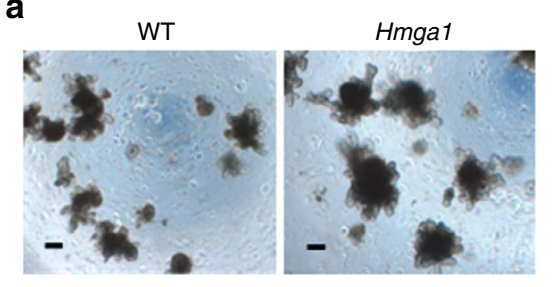

b

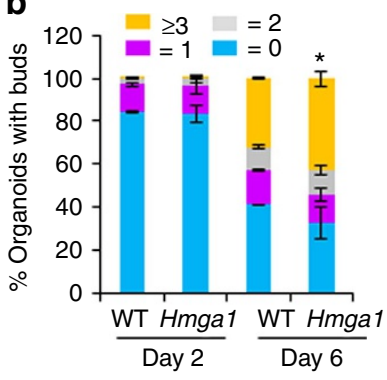

C

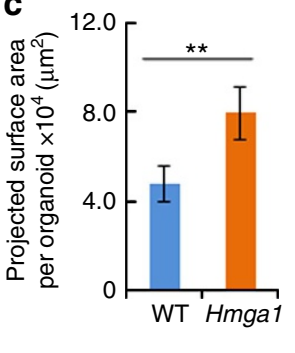

d
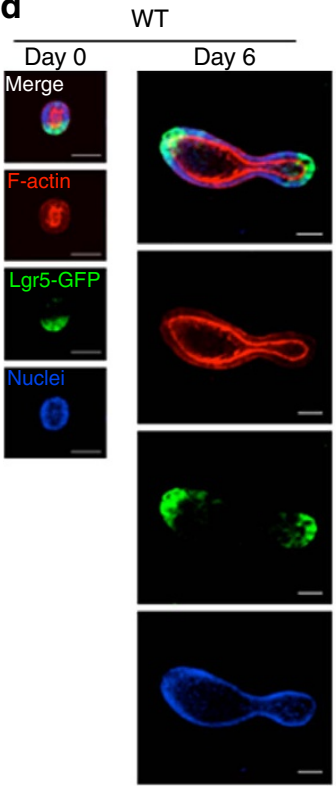

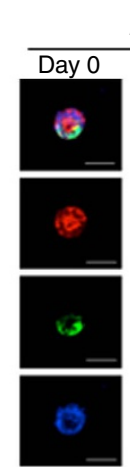

Hmga1

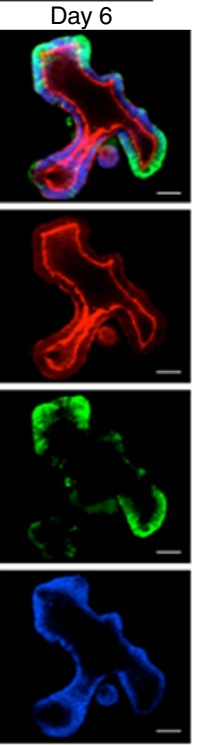

e

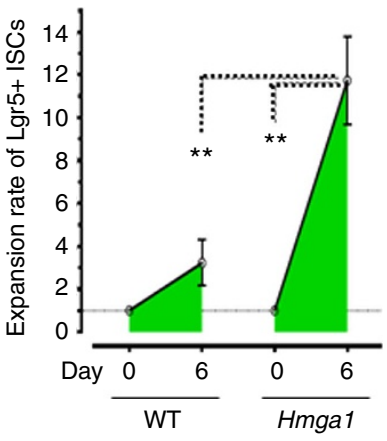

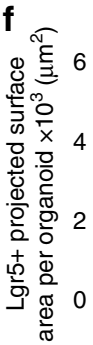

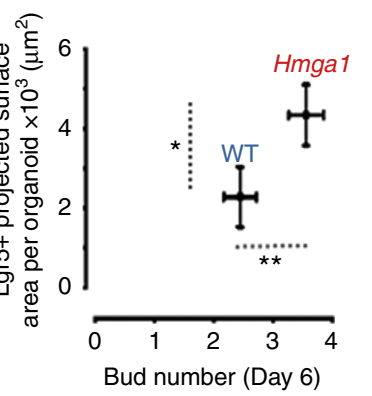

g

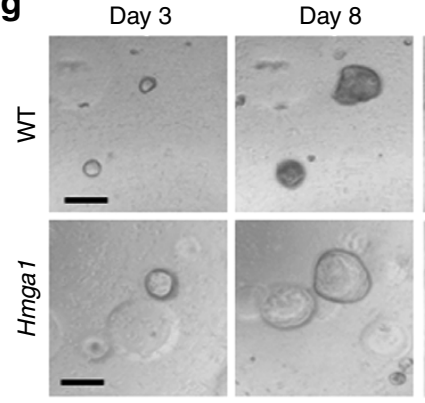

Day 11
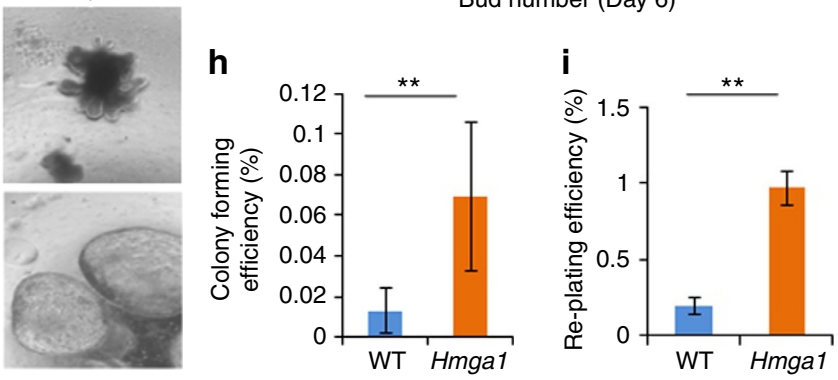

Figure 2 | Hmga1 enhances ISC function in gut organoid cultures. (a) Typical 3D organoids cultured from crypts are shown from WT or Hmga1 transgenic mice. (b) Bud numbers were ascertained in 3D organoids from isolated crypts ( $n>100$ organoids per mouse; 3 mice per genotype). Bars show mean percentage of organoids \pm s.d. with different bud numbers. (Yellow $\geq 3$, grey $=2$, pink $=1$, blue $=0$ ). The mean percentage of organoids with $\geq 3$ buds was increased in Hmgal organoids compared to WT organoids. ${ }^{\star} P<0.05$; two-tailed Student's $t$-test. (c) Organoid projected surface area (PSA) is shown (mean \pm s.d.) from WT or Hmga1 mice ( $n>20$ organoids/genotype). ${ }^{\star \star} P<0.01$; two-tailed Student's $t$-test. (d) Representative confocal imaging of crypt cells (day 0) and organoids (day 6) with GFP + staining for Lgr5 + ISCs, Phalloidin (F-actin; red) for cell clusters and organoid structure, and Hoechst (blue) for nuclei are shown. (e) Relative expansion rate of ISCs was calculated by the ratio of the PSA of GFP + ISCs on day 6 ( $n \geq 42$ organoids per group) over the PSA on day 0 ( $n \geq 72$ crypt cell clusters/group). ${ }^{\star \star} P<0.01$; Mann-Whitney test. (f) ISC PSA ( $y$ axis) and bud number ( $x$ axis) at days 0 and 6 are shown. ${ }^{\star \star} P<0.01,{ }^{\star} P<0.05$; Mann-Whitney test. (g) Lgr5-GFP + ISCs were purified by flow cytometry and cultured in matrigel. (h) Colony (organoid)-forming efficiency (mean \pm s.d.) was calculated from purified GFP + ISCs isolated as single cells $(n=3,000$ cells per group) from WT and Hmga1 mice in three experiments; ${ }^{\star} P<0.01$; two-tailed Student's $t$-test. (i) Re-plating efficiency (mean \pm s.d.; $n=3,000$ cells per group) was assessed after dissociating colonies from purified GFP + ISCs isolated as single cells from WT and Hmga1 mice using flow cytometry. ${ }^{\star \star} P<0.01$; two-tailed Student's t-test. Scale bars, $50 \mu \mathrm{m}$.

mice based on the projected surface area of Lgr5 + cells ( $\mu$ Manager software; Stanford Photonics; Fig. 2e,f). Similar to our prior results using standard microscopy, we also found more buds and greater projected surface area of Lgr5 + cells in the Hmgal organoids versus the WT organoids by confocal microscopy (Fig. 2f).
Previous work from our group demonstrated that forced expression of Hmgal prevents differentiation in human embryonic stem cells ${ }^{16}$. We therefore reasoned that Hmgal expression would predominate at the crypt-like regions enriched for ISCs at the bud tips and decrease in regions of differentiated cells at the 
base of the buds. To test this, we assessed Hmga 1 protein levels throughout the organoids and found that Hmgal is enriched at the bud tips where ISCs predominate, and undetectable in the differentiated cells, suggesting that differentiation is permitted in cells with lower levels of Hmgal (Supplementary Fig. 2). Together, these results suggest that Hmgal is a key factor for ISC maintenance and function.

Because we could not exclude the possibility that the enhanced stem cell function observed in the Hmgal organoids resulted from in vivo exposure to lymphoid or other cells with transgenic Hmgal expression and downstream factors, we engineered WT organoids to overexpress Hmgal. To this end, we transduced organoids via lentivirus expressing Hmgal and GFP (FUGWHmgal) and compared this to control organoids transduced with lentivirus expressing GFP alone (FUGW). The WT organoids engineered to overexpress Hmgal exhibited a similar phenotype to the organoids derived from the Hmgal mice with increased bud formation (Supplementary Fig. 3), demonstrating that this phenotype was dependent on Hmgal.

To determine whether the enhanced stem cell function in organoid-forming ability and bud development resulted from cell-autonomous properties of the Hmgal ISCs or from ISC interactions with other crypt epithelial cells, we purified Lgr5 + ISCs isolated as single cells from transgenic and WT mice using flow cytometry and compared their efficiency in organoid formation and re-plating assays. The purified Hmgal ISCs generated organoids at a fivefold greater efficiency as compared to WT ISCs (Fig. 2g,h). Moreover, re-plating efficiency was also enhanced fivefold in the Hmgal ISCs (Fig. 2i). Interestingly, the Hmgal organoids generated from purified Lgr5 + ISCs adopt a cyst-like, spherical structure similar to the morphology observed in organoids treated with Wnt or engineered to overexpress Ascl2, a transcription factor important in maintaining stem cell identity in ISCs ${ }^{7}$. To determine whether Hmgal organoids remained in a cyst-like structure because they were comprised predominantly of undifferentiated Lgr5 + stem cells, we stained for the ISC marker, Lgr5-GFP + . Strikingly, most of the cells within the Hmgal organoids generated from purified Hmga1 Lgr5-GFP + ISCs maintain this stem cell marker (Lgr5-GFP +; Supplementary Fig. 4), in contrast to WT organoids, which only stain for Lgr5GFP + at the tips of the buds (Fig. 2d). Together, these studies demonstrate that Hmgal maintains undifferentiated Lgr5 + ISCs and enhances self-renewal in this model.

Next, we sought to determine whether Hmgal is required for self-renewal and ISC function. First, we silenced Hmga1 in WT crypt cells using lentiviral-mediated delivery of short hairpin RNA (shRNA) targeting Hmgal (shHmgal) and compared this to WT crypt cells transduced with a control lentiviral vector in organoid cultures (Fig. 3a). Crypt cells were incubated with Wnt3a to enhance transduction efficiency as previously reported $^{2,3}$. Hmgal expression was repressed in the organoids transduced to express the shHmgal (Fig. 3b). Strikingly, the crypt cells with Hmgal silencing formed very few organoids with smaller projected surface areas and decreased bud number, while those transduced with control vector organized into typical 3D structures and generated new buds (Fig. 3a-c; Supplementary Fig. 5a). To rule out any potential nonspecific toxicity from the shHmgal vector, we also tested an inducible, shRNA lentiviral vector targeting Hmgal (inducible-shHmga1), which is linked to a gene encoding red fluorescent protein (RFP); both RFP and shHmgal are induced by doxycycline. Crypt cells transduced with the lentivirus, but not induced to express the shHmgal vector, organized into $3 \mathrm{D}$ organoids with buds similar to what we observed in WT crypt cultures (Fig. 3d; top panels). As expected, the uninduced organoids (cultured without doxycycline) do not express RFP. In contrast, those organoids in which the shHmgal vector was induced by doxycycline express $R F P$ and exhibit an impaired capability to proliferate and generate $3 \mathrm{D}$ organoids; the projected organoid surface area was decreased compared to uninduced controls (Fig. 3d,e). Bud formation was also disrupted (Supplementary Fig. 5b). Hmgal gene silencing in organoids transduced with the inducible shHmgal was confirmed by quantitative, reverse transcription PCR (qPCR; Fig. 3e). To further validate these findings, we transduced organoids derived from the Hmgal mice with the shHmgal vector and found that silencing Hmgal also disrupted their ability to form organoids and generate buds (Supplementary Fig. 5c). These studies indicate that Hmgal is a key factor involved in self-renewal, bud formation and organization into 3D organoid structures.

Hmga 1 induces self-renewal through Wnt/ $\mathbf{\beta}$-catenin signalling. When organoids from Hmgal mice were incubated with Wnt3a to enhance lentiviral transduction, we observed a striking phenotype whereby the Hmgal organoids formed very large, cystlike, spherical organoids comprised predominantly of Lgr5 + ISCs, in contrast to WT organoids, which generated smaller cysts at a decreased frequency (Fig. 4a; Supplementary Fig. 6). These findings suggested that the Hmgal organoids are more sensitive to Wnt signalling. Wnt/Tcf $4 / \beta$-catenin signalling is an evolutionarily conserved pathway important for self-renewal in epithelial crypt ISCs and many other tissue-specific adult stem cells ${ }^{38}$. Moreover, hyperactive Wnt signalling leads to malignant transformation in intestinal epithelium ${ }^{38}$. To begin to define the role of Hmgal in Wnt/Tcf4/ $\beta$-catenin signalling, we assessed immunostaining for $\beta$-catenin as a surrogate for canonical Wnt signalling. $\beta$-catenin was markedly increased in the Hmgal transgenic intestinal epithelium and concentrated at the base of the crypts (Fig. 4b). A similar increase was also observed in WT organoids transduced to overexpress Hmgal (Fig. 4c). Since Hmgal functions as an architectural transcription factor that alters gene expression, we hypothesized that Hmgal could upregulate expression of factors that enhance Wnt signalling. To test this, we first compared expression of genes encoding Wnt agonist receptors that function in intestinal epithelium, including Lgr5, Frizzled (Fzd)5, Fzd7, low-density lipoprotein receptorrelated protein 5 (Lrp5) and Lrp6 in purified Lgr5 + ISCs isolated from WT or transgenic mice. We found an increase in expression of all Wnt agonist receptors tested ( $\operatorname{Lgr} 5, \mathrm{Fzd} / \mathrm{H} / \mathrm{L}, \mathrm{Lr} 5 / 6)$ in the Hmgal ISCs by $>2$ to 4 -fold compared to control ISCs (Fig. 4d). Together, these results indicate that Hmgal amplifies Wnt signalling by upregulating genes encoding Wnt agonist receptors in ISCs, including the ISC marker, Lgr5, in our model.

Once $\beta$-catenin is released from an inhibitory complex following Wnt signalling, it binds to DNA together with its partner, Tcf4, to induce Wnt pathway genes. In human embryonic stem cells, HMGAl induces expression of $c-M Y C$ (ref. 16), a WNT/TCF4/ $\beta$-catenin gene target, suggesting that Hmgal could cooperate with Wnt/Tcf $4 / \beta$-catenin to regulate the Wnt stem cell program. We therefore assessed expression of Wnt/ Tcf $4 / \beta$-catenin target genes, including Axin2, Ascl2, $\beta$-catenin, Cd44, c-Myc, Ephb2, Ets, Tcf4 and Prom-1. Expression of all of these genes, excluding Prom-1, was increased by $>2$ to 6 -fold in Hmgal ISCs versus WT control ISCs (Fig. 4e). Together, these results indicate that Hmgal not only activates Wnt target genes together with Tcf4/ $\beta$-catenin, but also amplifies Wnt signals by inducing expression of Wnt agonist receptor genes.

To better define the role of Hmgal in regulating Wnt signalling, we cultured Hmgal organoids in the absence of the Wnt receptor agonist, R-spondin 1 (R-spo1), which is essential for organoid formation in this 3D culture system. R-spol is secreted by intestinal stromal cells and binds to the Lgr5 receptor 
a
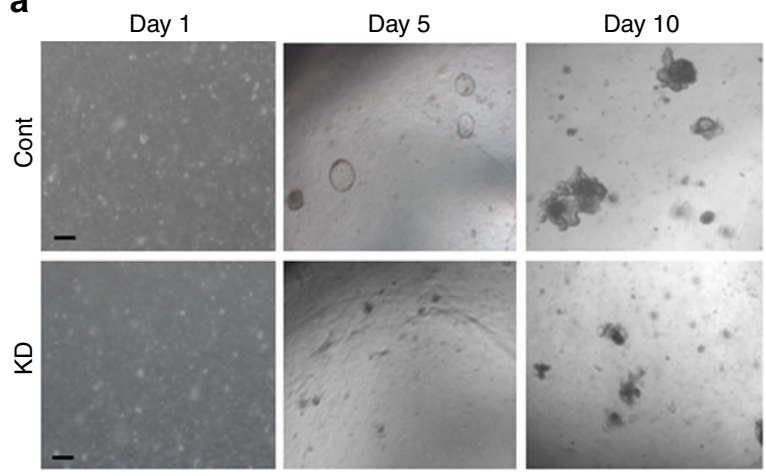

d

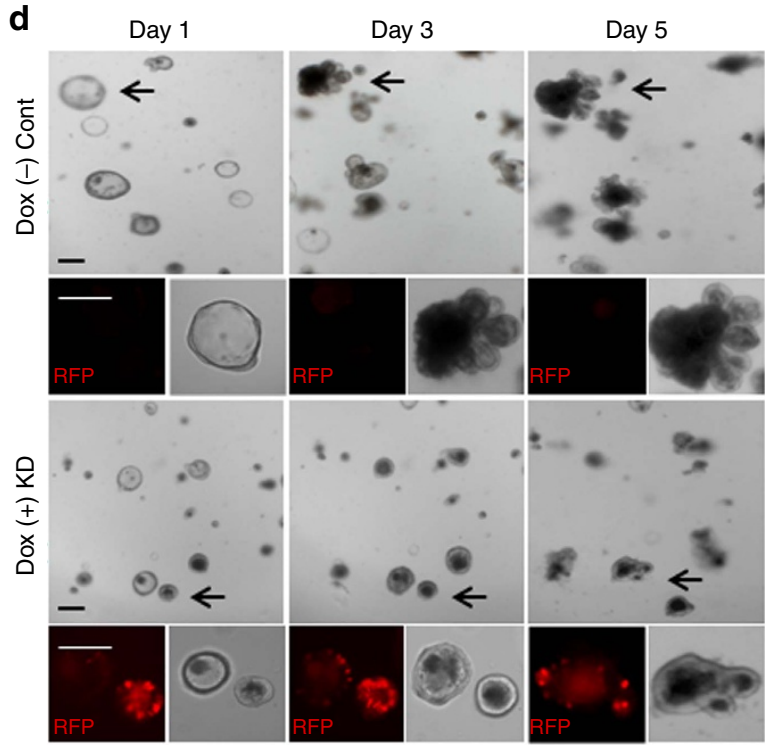

b
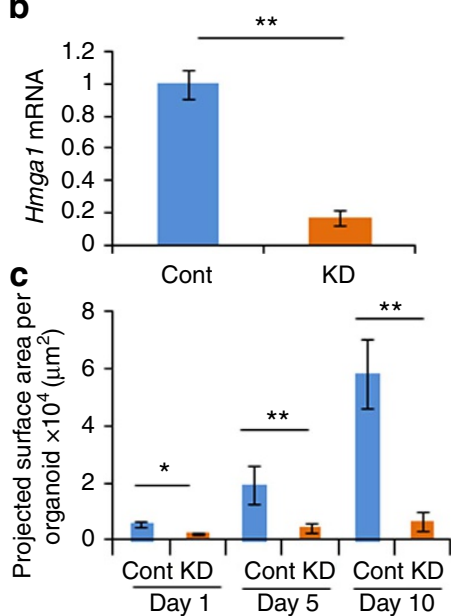

$\operatorname{Dox}(-)$ Cont
$\operatorname{Dox}(+) \mathrm{KD}$

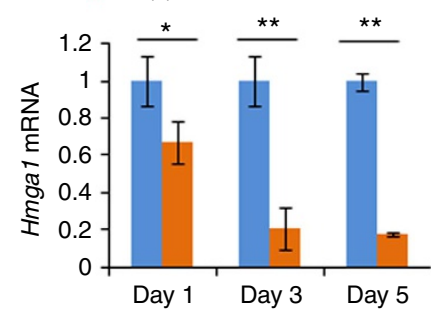

f

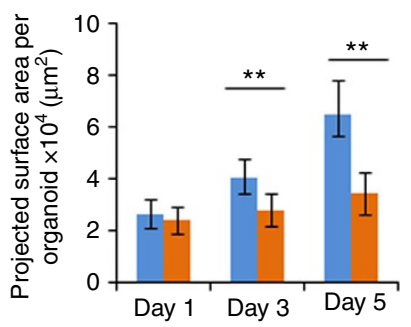

Figure 3 | Hmga1 deficiency disrupts ISC function in 3D organoids. (a) Representative images of organoid formation after silencing Hmga1 by delivery of lentiviral vector expressing shRNA targeting Hmgal (knockdown or KD) or control lentivirus (Cont) are shown. Scale bars, $50 \mu \mathrm{m}$. (b) Relative Hmga1 expression (mean \pm s.d.) was assessed from two experiments performed in triplicate (via qPCR) in organoids transduced with lentivirus (Cont versus KD) at day 10. Gapdh was used to control for loading. ${ }^{\star \star} P<0.01$; two-tailed Student's $t$-test. (c) PSA (mean \pm s.d.) was assessed in organoids transduced with lentivirus (Cont versus KD) at the indicated time points ( $n=20$ organoids per group). ${ }^{\star} P<0.05,{ }^{\star \star} P<0.01$; two-tailed Student's $t$-test.

(d) Representative image of organoid formation \pm knockdown of Hmgal via an inducible lentiviral vector expressing red fluorescent protein (RFP) and shRNA targeting Hmgal are shown. Doxcyline $\left(0.5 \mu \mathrm{g} \mathrm{ml}^{-1}\right.$ ) was used to induce RFP and shRNA. Scale bars, $50 \mu \mathrm{m}$. (e) Relative Hmgal expression (mean \pm s.d.) in organoids with or without induction of lentivirus shRNA targeting Hmgal was assessed from two experiments performed in triplicate (via qPCR); Gapdh was used to control for loading. ${ }^{\star} P<0.05,{ }^{\star \star} P<0.01$; two-tailed Student's $t$-test. (f) PSA (mean \pm s.d.) of organoids with or without induction of Hmga1 knockdown at the indicated time points are shown $\left(n=20\right.$ organoids per group from two different virus transduction experiments). ${ }^{\star \star} P<0.01 ;$ Mann-Whitney test.

to activate Wnt signalling ${ }^{39,40}$. We discovered a striking difference in the response of Hmgal organoids to the absence of R-spol as compared to the WT controls: Hmgal organoids continued to survive and proliferate for over 2 weeks, while the WT organoids lost their 3D structural organization and ultimately died by day 5 (Supplementary Fig. 7). By 3 weeks, however, the Hmgal organoids also stopped proliferating and lost their 3D organization, suggesting that Hmgal overexpression partially rescues loss of Wnt signalling via R-spo1, but does not completely recapitulate or bypass Wnt.

To further investigate the link between HMGA1 and Wnt, we determined whether cells overexpressing HMGA1 have enhanced Wnt reporter activity using an established Wnt reporter construct that includes seven canonical Tcf $4 / \beta$-catenin-binding sites in tandem upstream of a luciferase reporter gene ${ }^{46}$. Both HEK 293 human embryonal kidney cells and Caco-2 colon cancer cells were transduced with the Wnt reporter lentiviral vector, which includes a puromycin resistance gene. After selection for stable integration of the Wnt reporter construct by puromycin, the cultured cells were transduced with either control retrovirus or retrovirus expressing human HMGA1. In both cell types, HMGA1 activated Wnt reporter activity, while retroviral delivery of the control virus failed to do so (Fig. 4f). These experiments further support the role of HMGA1 in activating Wnt signalling.

To further test the link between Hmgal and Wnt, we treated crypt cells with the Wnt inhibitor, C59, which blocks Wntmediated transcription and cell proliferation by inhibiting porcupine (PORCN), a protein required for Wnt palmitoylation, secretion and biological activity. Crypt cells isolated from Hmgal or WT small intestine were selected for small crypt size and cell number by passage through a $40 \mu \mathrm{M}$ filter. The Hmgal crypts 
a

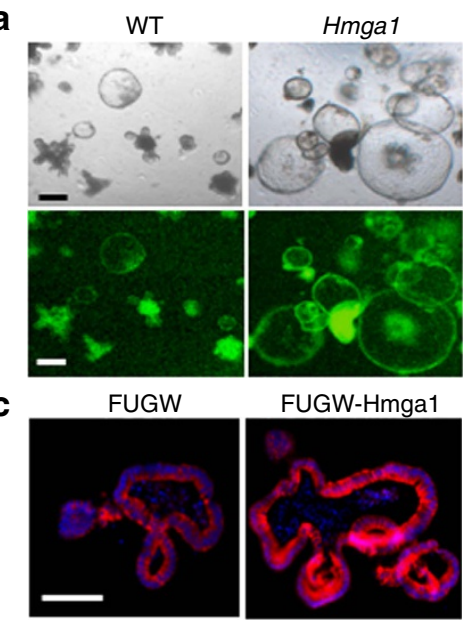

d

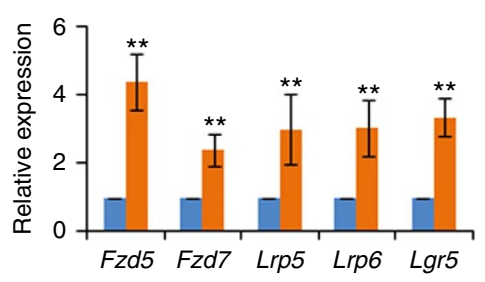

b
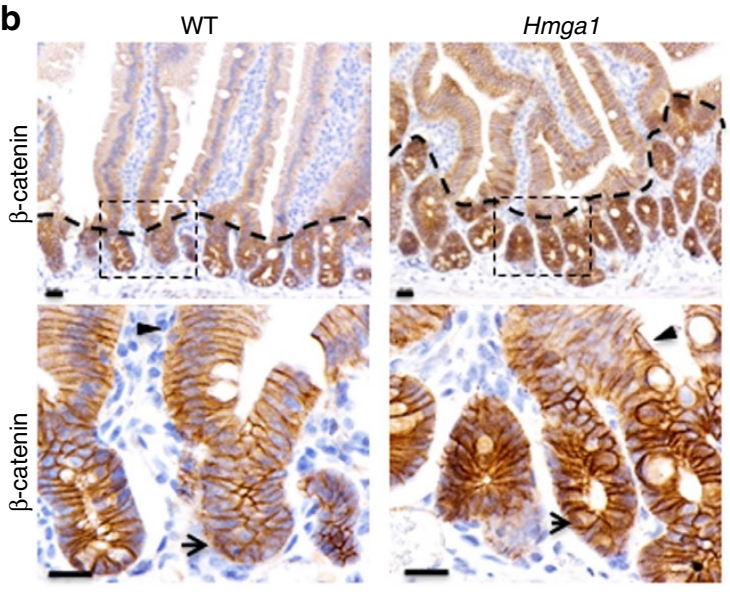

e

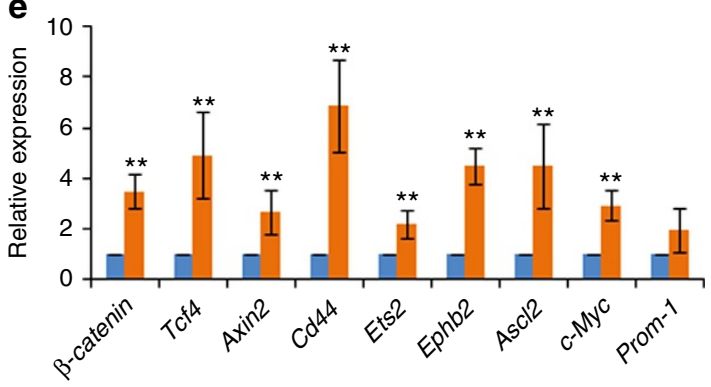

f
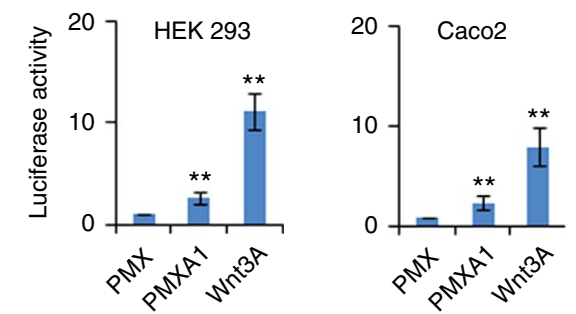

g

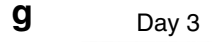

Day 3

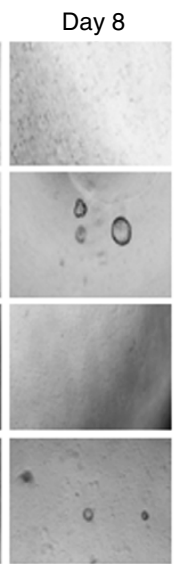

Day 14

h
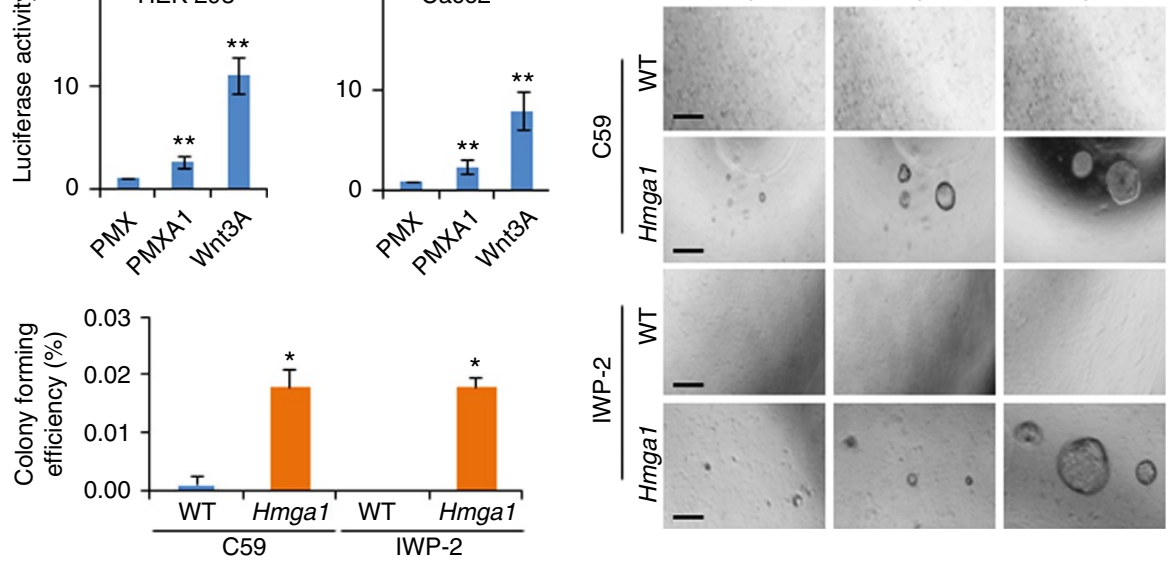

Figure 4 | Hmga1 amplifies Wnt signalling in ISCs. (a) Representative organoids after exposure to Wnt3a (10 ng $\left.\mu^{-1}\right)$ are shown. Hmgal organoids form large, cyst-like spheres (standard (top) and fluorescent (bottom) microscopy). Scale bars, $50 \mu \mathrm{m}$. (b) $\beta$-Catenin IHC staining is shown in small intestines from WT and Hmgal mice. Scale bars, $20 \mu \mathrm{m}$. (c) $\beta$-Catenin IHC staining is shown in organoids engineered to express control lentivirus (FUGW; left) or Hmgal (FUGW-Hmga1; right). Scale bar, $20 \mu \mathrm{m}$. (d) Relative expression (mean \pm s.d.) of genes encoding Wnt agonist receptors was ascertained by qPCR in GFP + ISCs from WT (blue) or Hmga1 transgenic mice (orange) in two experiments performed in triplicate. Gapdh was used to control for loading. ${ }^{\star \star} P<0.01$; two-tailed Student's $t$-test. (e) Relative expression (mean \pm s.d.) of genes encoding Wnt/Tcf $4 / \beta$-catenin transcriptional targets was ascertained by qPCR in GFP + ISCs from WT or Hmga1 transgenic mice in two experiments performed in triplicate. Gapdh was used to control for loading. ${ }^{\star \star} P<0.01$; two-tailed Student's t-test. (f) HEK 293 (left) and Caco2 (right) cells infected with the synthetic Wnt reporter construct containing seven optimal Tcf4/ $\beta$-catenin-binding sites showed activation in cells overexpressing Hmgal. Purified Wnt3A (100 ng ml $\left.{ }^{-1}\right)$ protein was used as a positive control. Bars show mean luciferase activity \pm s.d. from two experiments performed in triplicate. ${ }^{\star \star} P<0.01$; two-tailed Student's $t$-test. (g) Representative image of GFP + ISCs from WT or Hmga1 mice are shown in 3D culture with Wht porcupine inhibitors IWP-2 (0.5 $\mu$ M) and C59 (0.5 $\mu$ M). Scale bars, $50 \mu$ m. (h) Relative colony-forming efficiency (mean \pm s.d.) from three experiments is shown with GFP + ISCs from WT or Hmga1 mice in 3D culture with the Wnt porcupine inhibitors (IWP-2, C59). ${ }^{\star} P<0.05$; two-tailed Student's $t$-test.

generated organoids and buds in C59 $(0.25 \mu \mathrm{M})$ with a greater projected surface area than WT crypt cells cultured with C59 (Supplementary Fig. 8). In addition, the mean projected surface area of WT organoids cultured in C59 was reduced by days 6 and 10 compared to WT organoids cultured under control conditions with vehicle alone. In contrast, the Hmgal crypt cells were relatively impervious to $\mathrm{C} 59$; by day 6 , there was no difference in projected surface area of Hmgal organoids compared to those cultured with vehicle alone, although the projected surface area decreased by day 10 when cultured with C59 compared to those 
cultured with vehicle control. This blunted response of Hmgal organoids to Wnt inhibition could result from the ability of Hmgal to amplify Wnt signalling, thereby mitigating the effects of Wnt inhibition. Alternatively, or in addition to amplifying Wnt signalling, the Hmgal organoids could dampen the effects of Wnt inhibition by providing a buffer as a result of an increased size and cell number. Finally, an increase in the number of Paneth cells, which secrete Wnt agonists, could mitigate the effects of Wnt inhibition. To distinguish between these possibilities, we isolated single, purified Lgr5 + ISCs from transgenic or WT mice and cultured them in the presence of two different Wnt PORCN inhibitors, C59 and IWP-2. Strikingly, the Hmgal ISCs proliferated and formed 3D, cyst-shaped organoids for up to 10 days, albeit at a slower rate between days 6-10 compared to purified Hmgal ISCs cultured without inhibitor (Fig. 4g,h). In contrast, the WT ISCs generated very few organoids when cultured with C59. Moreover, no organoids formed from WT ISCs cultured in IWP-2 (Fig. 4g,h). Together, these findings demonstrate that Hmgal ISCs have a blunted response to Wnt inhibitors, which is consistent with their ability to amplify Wnt signals.

Hmgal expands the Paneth cell niche and upregulates Sox9. Paneth cells are terminally differentiated epithelial cells derived from ISCs and located at the base of intestinal crypts ${ }^{47-50}$. They support ISC survival by secreting Wnt3a and other factors, thus providing an epithelial niche for ISCs. To determine whether Hmgal alters the Paneth cell niche in our transgenic models, we stained for lysozyme using alkaline phosphatase, which marks Paneth cells. There was a marked increase in lysozyme stain (Fig. 5a), which was confirmed quantitatively by immunohistochemistry (IHC) pixels (Fig. 5b). To determine whether the increase in lysozyme stain corresponds to an increase in Paneth cell number, Paneth cells were enumerated in mouse intestinal tissue after controlling for the region of the intestine (Fig. 5c; $n=100$ crypts per group from duodenal epithelium). There was a marked increase in Paneth cell number per crypt, which paralleled the increase in lysozyme stain by IHC. Next, we co-stained the duodenal epithelium with EpCAM to demarcate cell borders, DAPI to indicate individual nuclei, and lysozyme such that the frequency of cells staining positive for lysozyme could be enumerated within the crypts (Fig. 5d). There was also a marked increase in Paneth cell frequency (Fig. 5e; $n=50$ crypts per group from duodenal epithelium), consistent with an expansion in the Paneth cell niche in the Hmgal transgenic duodenal epithelium as compared to WT control duodenal epithelium. To further confirm the increase in Paneth cells by Hmgal, we assessed Paneth cell number in Hmgal organoids using two different approaches. First, Paneth cells have a distinctive granular appearance on phase contrast microscopy. We therefore compared the mean number of granular cells per bud in WT and Hmgal organoids after controlling for bud size by dividing the Paneth cell number by the projected surface area per bud (Fig. 5f,g; $n=35$ buds per group). Similar to the Hmgal mouse intestinal tissue, there was a significant $(P<0.00001$; Mann-Whitney test) increase in Paneth cell number per bud projected surface area in the Hmgal organoids (Fig. 5g). Second, we assessed Paneth cell number by staining with lysozyme, which appeared to be increased in organoids transduced to express Hmgal compared to control organoids (Fig. 5h). To quantitatively assess the frequency of Paneth cells per total bud cell number, we co-stained with EpCAM, DAPI and lysozyme. There was an increase in Paneth cell frequency using this approach, similar to our results with intestinal tissue and granular cell number (Fig. 5h,i). Finally, to further corroborate this result, we assessed expression of a transcript that is highly specific for
Paneth cells, Defcr-rs ${ }^{51}$, and found that it is increased in the Hmgal organoids compared to control organoids (Fig. 5j). Together, these results indicate that Hmgal induces Paneth cell niche formation and expansion, which could help to promote ISC maintenance and expansion.

Because terminal differentiation to a Paneth cell requires Sox9 (refs 47-49), we hypothesized that Hmgal fosters Paneth cell expansion by upregulating Sox 9 expression. HMGA1 induces the SOX family member, SOX2, in human embryonic stem cells ${ }^{16}$; further, both human and mouse $S O X 9 /$ Sox 9 have similar AT-rich regions and predicted Hmgal DNA-binding sites in the $5^{\prime}$ untranslated region. Sox 9 is also a $\beta$-catenin/Tcf 4 target gene. We found that Hmgal upregulates Sox9 expression in the Lgr5+ ISCs as compared to WT Lgr5 + ISCs (Fig. 6a). To determine whether Hmgal directly induces Sox 9 gene expression, we performed chromatin immunoprecipitation (ChIP) in intestinal crypt cells from WT mice. We discovered that Hmgal binds directly to the Sox9 promoter at two conserved sites (Fig. 6b; Supplementary Fig. 9a,b). Enrichment for Hmgal binding was greatest at the proximal site (denoted site 1) and significant, albeit lower, at the second most proximal site (site $2 ; P<0.01$ by twotailed Student's $t$-test). Hmgal binding was not enriched at the distal site (site 3 ). For a positive control, we used a histone H3 antibody, which showed significant enrichment $(P<0.01$ by twotailed Student's $t$-test) at all promoters tested. For a negative control, we used the IgG antibody, which had minimal binding to chromatin at all promoter regions tested. As a second negative control, we used the murine Hprt gene; this promoter region was previously shown to be negative for Hmgal occupancy. We confirmed that Hmgal does not occupy the Hprt promoter (Fig. 6b). Next, we performed transfection experiments to determine whether Hmgal transactivates the Sox9 promoter reporter construct containing the Hmgal-binding site 1, which had the greatest enrichment for Hmgal binding (Supplementary Fig. 9a-c). As a control, we included a Sox9 promoter reporter construct lacking this Hmgal-binding site (Supplementary Fig. 9b,c). We found that Hmgal induced the Sox 9 promoter construct that included site 1 , while there was no induction of the promoter construct lacking this site (Supplementary Fig. 9d). As an additional control, we included a dominant-negative HMGA1 construct with mutations in the second AT-hook DNA-binding domain, which no longer binds to $\mathrm{DNA}^{16}$. The dominantnegative mutant also failed to transactivate either Sox9 promoter construct (Supplementary Fig. 9d). To further validate this relationship between Hmgal and Sox9, we assessed Sox9 mRNA in organoid cells transduced to overexpress Hmgal and found that Sox9 was induced by Hmgal (Supplementary Fig. 9e). In contrast, we also found that Sox 9 was repressed in organoids with Hmgal knockdown following transduction with shRNA targeting Hmgal compared to controls (Supplementary Fig. 9f).

To determine whether cells with high levels of Hmgal protein also have high levels of Sox9 protein, we assessed Sox9 protein and found that it is increased in the crypts of the Hmgal transgenic mice compared to WT controls by IHC (Fig. 6c). To quantitatively assess Hmgal and Sox9 proteins, we performed western blot analysis of crypt cells from WT and transgenic mice; the Hmgal transgenic crypt cells had an increase in both Hmgal and Sox9 protein compared to WT crypt cells (Fig. 6d,e). Next, we compared adjacent sections stained for Hmga1 or Sox9 and found that the majority of cells with detectable Hmgal at the base of the crypts also have detectable Sox9 protein in both WT and transgenic mice (Fig. $6 \mathrm{f}, \mathrm{g}$ ). In WT mice, $>70 \%$ of cells that stained positive for Hmgal also stained positive for Sox9. In the transgenic mice, which have more cells with Hmgal staining and higher levels of Hmga 1, we found that $>95 \%$ of cells that stained positive for Hmgal also stained positive for Sox9 (Fig. 6f,g). 


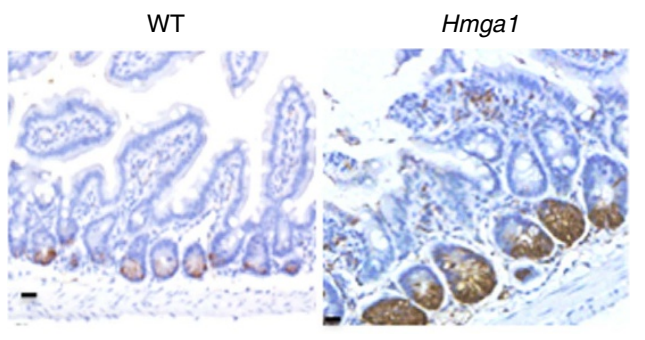

b

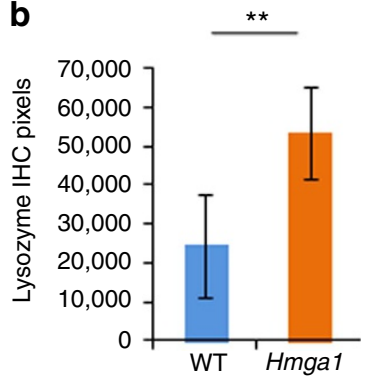

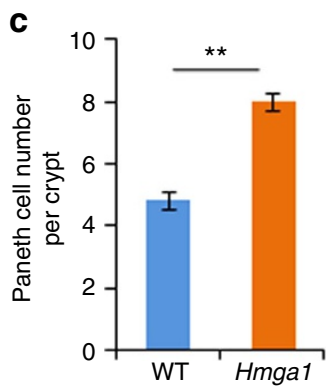

f
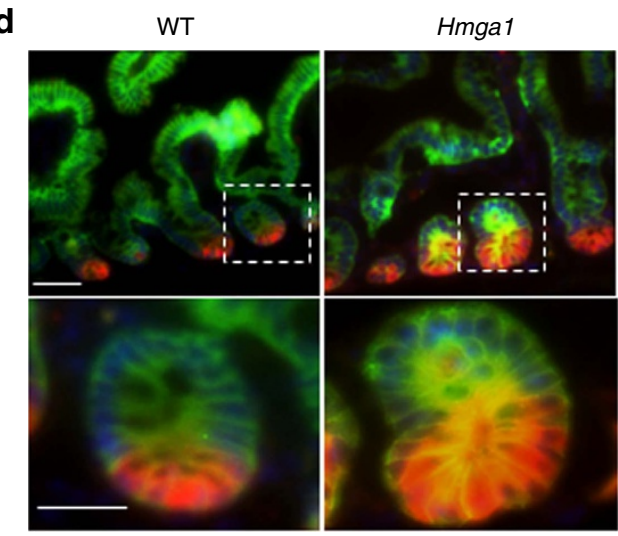

e

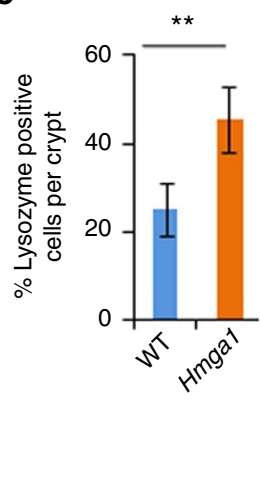

W

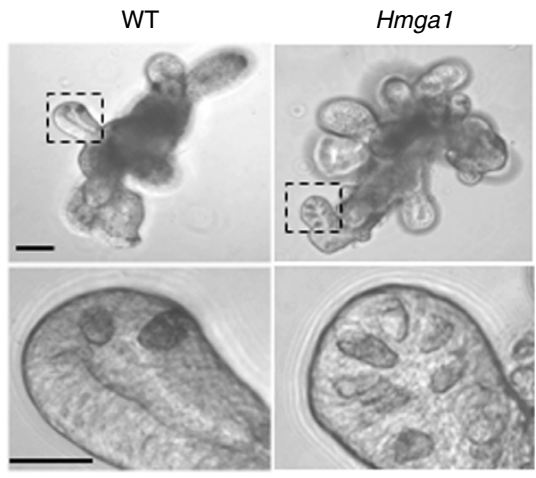

i

j

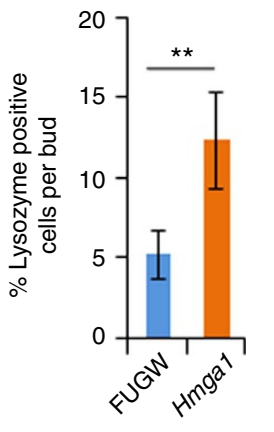

Figure 5 | Hmga1 expands the Paneth cell niche. (a) IHC for lysozyme is shown from small intestines of WT and Hmga1 transgenic mice. Scale bar, $20 \mu \mathrm{m}$. (b) Lysozyme IHC pixels (mean \pm s.d.; imagine pro-plus 6.0 software) is shown in small intestinal cross-sections ( $n=78$ crypts per group; 3 mice per genotype). ${ }^{\star \star} P<0.01$; Mann-Whitney test. (c) Paneth cell number (mean \pm s.d.) per crypt $\left(n=100\right.$ crypts per group; 3 mice per genotype). ${ }^{\star \star} P<0.00001$; Mann-Whitney test. (d) Immunofluorescent co-staining for lysozyme, Ep-CAM and DAPI is shown from small intestines of WT and Hmga1 transgenic mice. Scale bars, $20 \mu \mathrm{m}$. (e) Paneth cell frequency (mean \pm s.d.) was obtained by dividing the Paneth cell number by total cell number per crypt in WT and Hmga intestine ( $n=50$ crypts per group; 3 mice per genotype). ${ }^{\star *} P<0.00001$; Mann-Whitney test. (f) Granular cells in WT and Hmga1 organoid buds are shown via phase contrast microscopy. Scale bars, $50 \mu \mathrm{m}$. (g) Granular cells per bud PSA (mean \pm s.d.) from WT and Hmga1 organoids are shown ( $n=35$ buds per group). ${ }^{\star \star} P<0.00001$; Mann-Whitney test. (h) IHC for lysozyme (top) and immunofluorescent co-staining for lysozyme, Ep-CAM and DAPI (bottom) are shown in organoids from WT or Hmga1 mice. Scale bars, $50 \mu \mathrm{m}$. (i) Paneth cell frequency per bud (mean \pm s.d.) was calculated by dividing the Paneth cell number by the total bud cell number ( $n=30$ organoids per group). ${ }^{\star \star} P<0.00001$; Mann-Whitney test. (j) Relative expression (mean $\pm \mathrm{s} . d$.) of Defcr-rs, the Paneth cell-specific transcript, is shown from two experiments performed in triplicate from organoids from WT and Hmga1 mice. Gapdh was used to control for loading. ${ }^{\star \star} P<0.01$; two-tailed Student's $t$-test.

While Sox9 is required for Paneth cell differentiation ${ }^{4-51}$, it is not known whether increased levels of Sox9 lead to expansion in Paneth cells. To test this, we transduced WT organoids with a lentivirus expressing Sox9 following induction by doxycycline (Fig. 7). Immunofluorescent staining showed increased Sox9 protein in the doxycycline-induced organoids compared to uninduced control organoids cultured for the same period of time (Fig. 7a). Sox9 protein (by western blot analysis) and mRNA (by qPCR) were both increased relative to uninduced organoids (Fig. $7 b, c)$. Consistent with the observation that Sox 9 decreases proliferation in vivo in intestinal epithelium ${ }^{49}$, we observed that the organoid growth (estimated by projected surface area) was decreased in organoids with Sox9 induction compared to uninduced organoids (Fig. 7d,e). We also found that the projected bud surface area was decreased with Sox9 induction (Fig. 7f). Next, we compared Paneth cell frequency in Sox9induced and uninduced organoids using two different approaches. First, we used phase contrast microscopy to identify Paneth cells morphologically by the distinctive granules, which are characteristic of these cells. The relative 

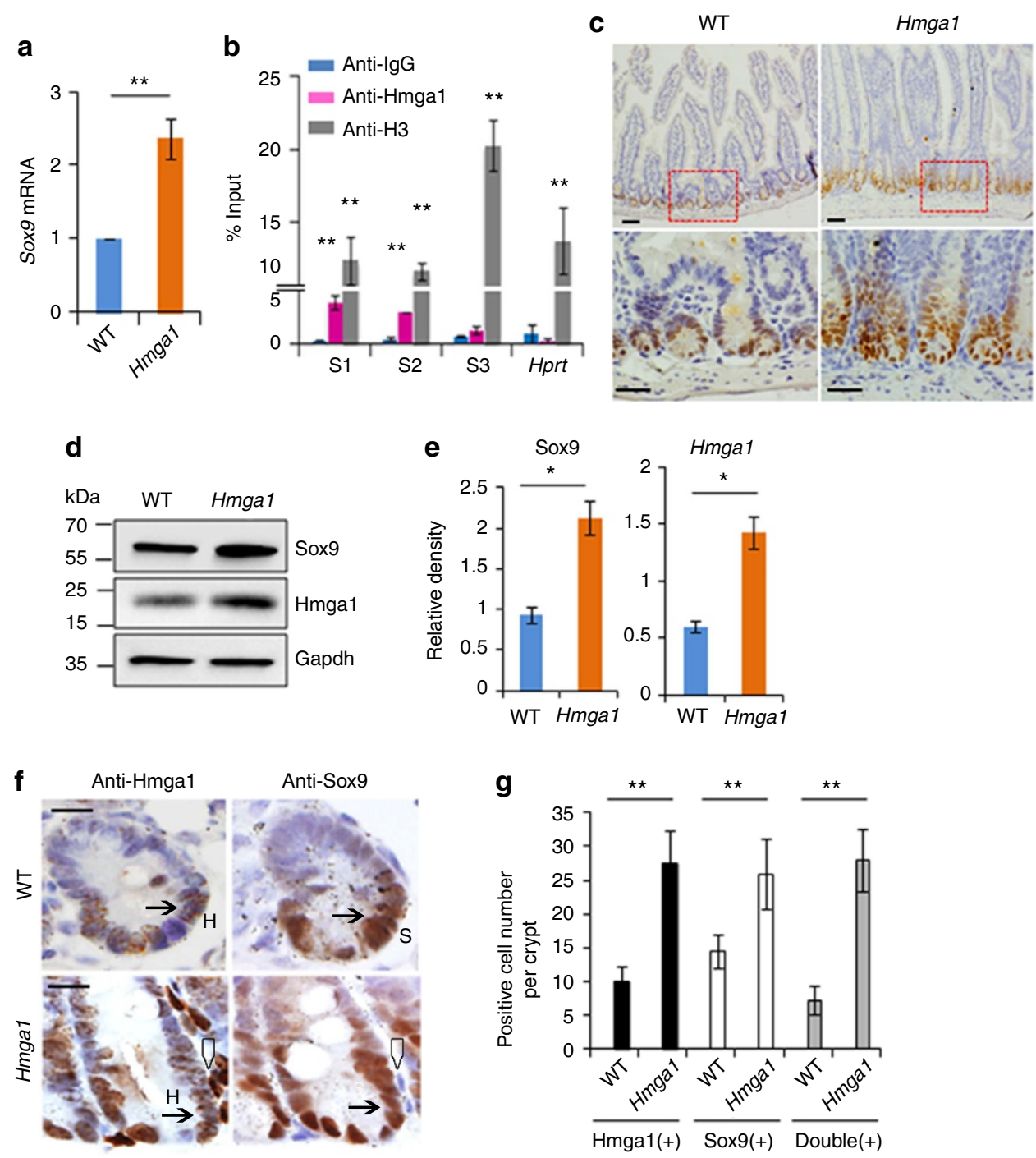

Figure 6 | Hmga1 directly induces Sox9 expression. (a) Relative Sox9 mRNA (mean \pm s.d.) by qPCR in GFP ${ }^{+}$ISCs from WT and Hmga1 mice is shown from two experiments performed in triplicate. Gapdh was used to control for loading. ${ }^{\star \star} P<0.01$; Student's $t$-test. (b) Hmga1 binding to the Sox9 promoter by ChIP is shown from mouse crypt cells. IgG antibody and the Hprt promoter region were used as negative controls; histone $\mathrm{H} 3$ was a positive control. Bars show mean enrichment \pm s.d. from two experiments performed in triplicate; ${ }^{\star \star} P<0.01$; two-tailed Student's $t$-test. (c) Sox 9 IHC staining (brown) is shown in intestinal cross-sections from WT and Hmga1 transgenic mice. Scale bars, $20 \mu \mathrm{m}$. (d) Western blots for Sox9, Hmga1, and Gapdh (loading control) were performed from freshly isolated crypt cells from WT and Hmga1 transgenic mice. Western blots were done three times; a representative blot is shown. Size markers $(\mathrm{kDa})$ are indicated. (e) Densitometry analysis $\pm \mathrm{s}$.d. was performed on repeat western blots. ${ }^{\star} P<0.05$; two-tailed Student's $t$-test. (f) Sox 9 and Hmga1 IHC staining (brown) is shown in adjacent sections of small intestine from WT and Hmga1 mice. Arrows show representative staining for Hmga1 (H) or Sox9 (S); triangles show Hmga1 transgenic lymphocytes which have high levels of Hmga1 (but not Sox9). Scale bars, $50 \mu \mathrm{m}$. (g) Mean number of cells \pm s.d. staining positive for Hmga1, Sox9 or both were identified by IHC in adjacent cross-section on slides of small intestinal crypts from WT and Hmgal mice ( $n \geq 21$ crypts per group.) ${ }^{\star \star} P<0.01$ by Mann-Whitney test.

Paneth cell number per bud-projected surface area was increased in the Sox9-induced organoids compared to uninduced control organoids (Fig. 7g,h). Second, we used lysozyme stain to identify Paneth cells, together with EpCAM stain (to delineate cell membranes) ${ }^{50}$ and DAPI (to demarcate nuclei). There was also an increase in the frequency of Paneth cells per total bud cell number in Sox9-induced organoids $(n>50$ buds per condition from $>50$ organoids per condition; Fig. 7i,j). To further substantiate these results, we assessed expression of a transcript that is highly specific for Paneth cells, Defcr-rs ${ }^{51}$, and showed that it is also increased in the organoids overexpressing Sox9 (Fig. 7k). These results suggest that Hmgal-mediated induction of Sox9 helps to expand the Paneth cell niche, although there are likely to be other factors that contribute to Paneth cell differentiation. Together, our results demonstrate that Hmgal drives self-renewal and expansion of ISCs in addition to helping to establish an epithelial stem cell niche through Paneth cell differentiation.

HMGA1 and SOX9 in colonic epithelium and colorectal cancer. To determine whether our findings in mouse intestine are relevant to humans, we assessed expression of HMGA1 and SOX9 in human intestinal epithelium. Using the Cancer Genome Atlas (TCGA), we found that HMGA1 and SOX9 are positively correlated in normal colonic epithelium $(P=0.008, r=0.52$; Fig. 8a). We also stained human small intestine, and found that HMGA1 localizes to the columnar basal cells within the crypts where ISCs 

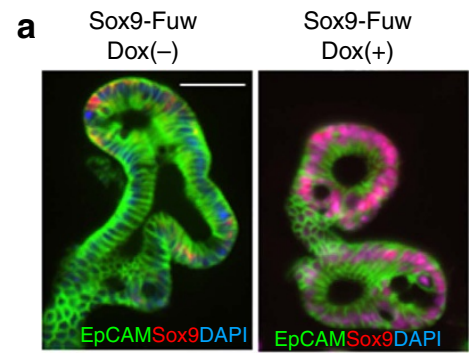

d Sox9-Fuw

Sox9-Fuw

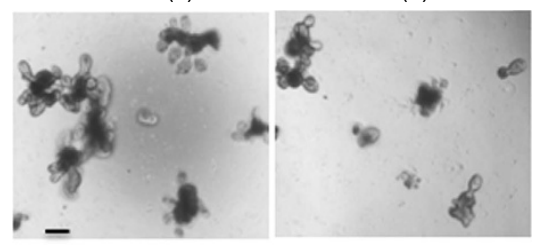

g
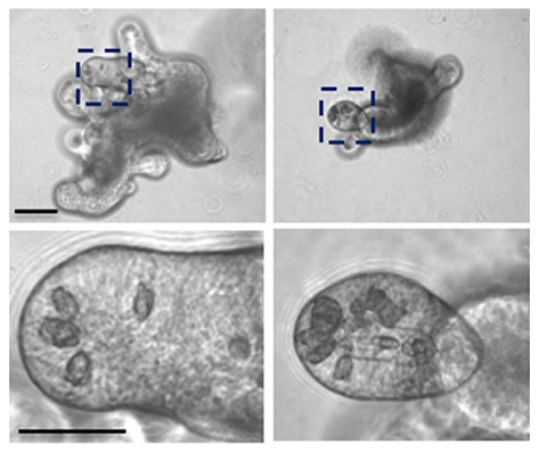

i

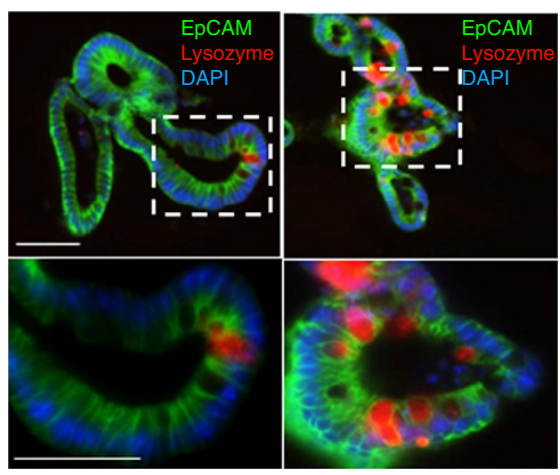

b

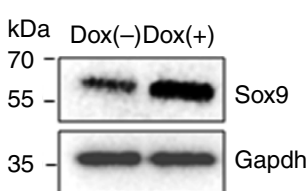

Gapdh

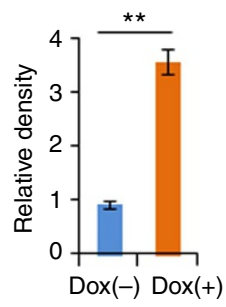

C

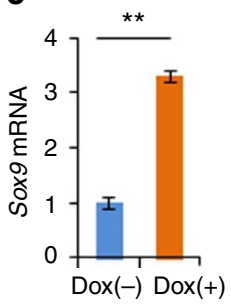

e

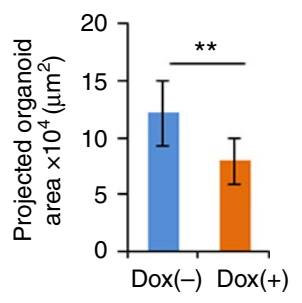

f

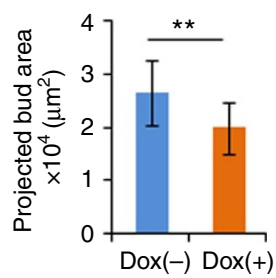

h

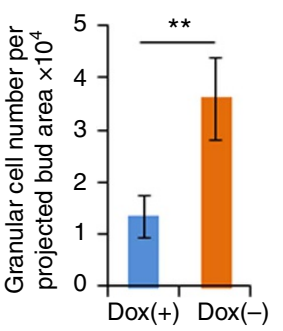

j

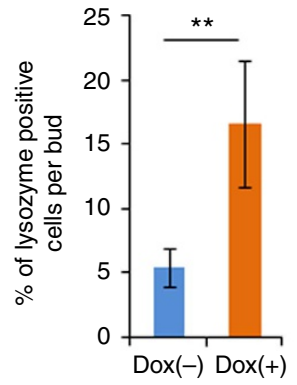

k

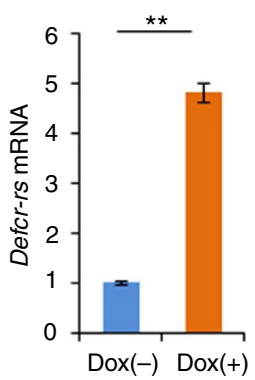

Figure 7 | Paneth cell number increases in organoids overexpressing Sox9. (a) Immunofluorescent stain for Sox9 (red), DAPI (blue) and EpCAM (green) is shown in organoids transduced with lentivirus expressing doxycycline-dependent inducible Sox9 (Sox9-Fuw). Sox9 (Sox9-Fuw Dox( + )) was induced by doxycycline $\left(1 \mu \mathrm{g} \mathrm{ml}^{-1}\right)$ for 5 days; controls were cultured for 5 days without doxycycline. (b) Representative western blot for Sox 9 in control and induced organoids is shown. Densitometry \pm s.d. was performed on three repeat western blots. ${ }^{\star \star} P<0.01$; two-tailed $t$-test. (c) Sox 9 mRNA (mean \pm s.d.) was assessed by qPCR from two experiments performed in triplicate in control and induced organoids. Gapdh was used to control for loading. ${ }^{\star \star} P<0.01$; twotailed Student's t-test. (d) Typical organoids from uninduced controls (left) and induced (right) cultures are shown. (e) PSA \pm s.d. of organoids ( $n=52$ per group) are shown. ${ }^{\star \star} P<0.01$; two-tailed Student's $t$-test. (f) PSA \pm s.d. of buds ( $n=52$ per group) are shown. ${ }^{\star \star} P<0.01$; two-tailed Student's $t$-test. (g) Paneth cells identified as granular cells using phase contrast microscopy within individual buds are shown. (h) Granular cell frequency (mean \pm s.d.) was estimated by dividing the total granular cell number by the total bud PSA ( $n=52$ per group). ${ }^{\star \star} P<0.01$; Mann-Whitney test. (i) Paneth cells were identified based on lysozyme stain (red) in cells delineated by EpCAM (green) for cell membrane and (DAPI) for nuclei. (j) Paneth cell frequency (mean \pm s.d.) was ascertained by dividing the number of lysozyme staining cells (red) delineated by EpCAM (green) by the total cell number (DAPI) per bud ( $n=35$ per group). ${ }^{\star \star} P<0.01$; Mann-Whitney test. (k) Relative expression of Defc-rs (mean \pm s.d.) was compared in uninduced control and Sox9-induced organoids by qPCR from two experiments performed in triplicate. Gapdh was used to control for loading. ${ }^{\star \star} P<0.01$; two-tailed Student's $t$-test. Scale bars, $50 \mu \mathrm{m}$. 
a

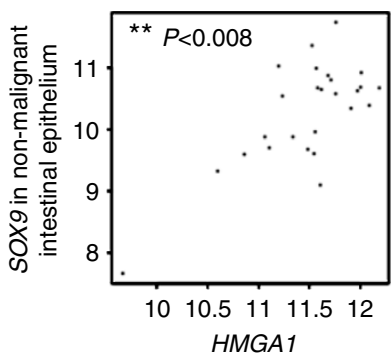

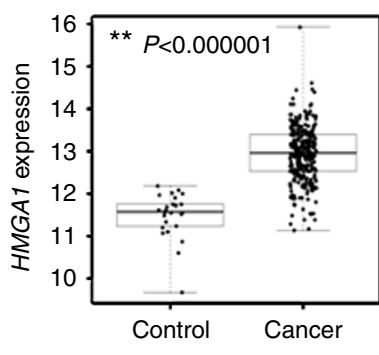

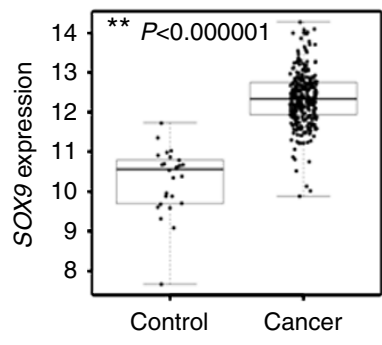

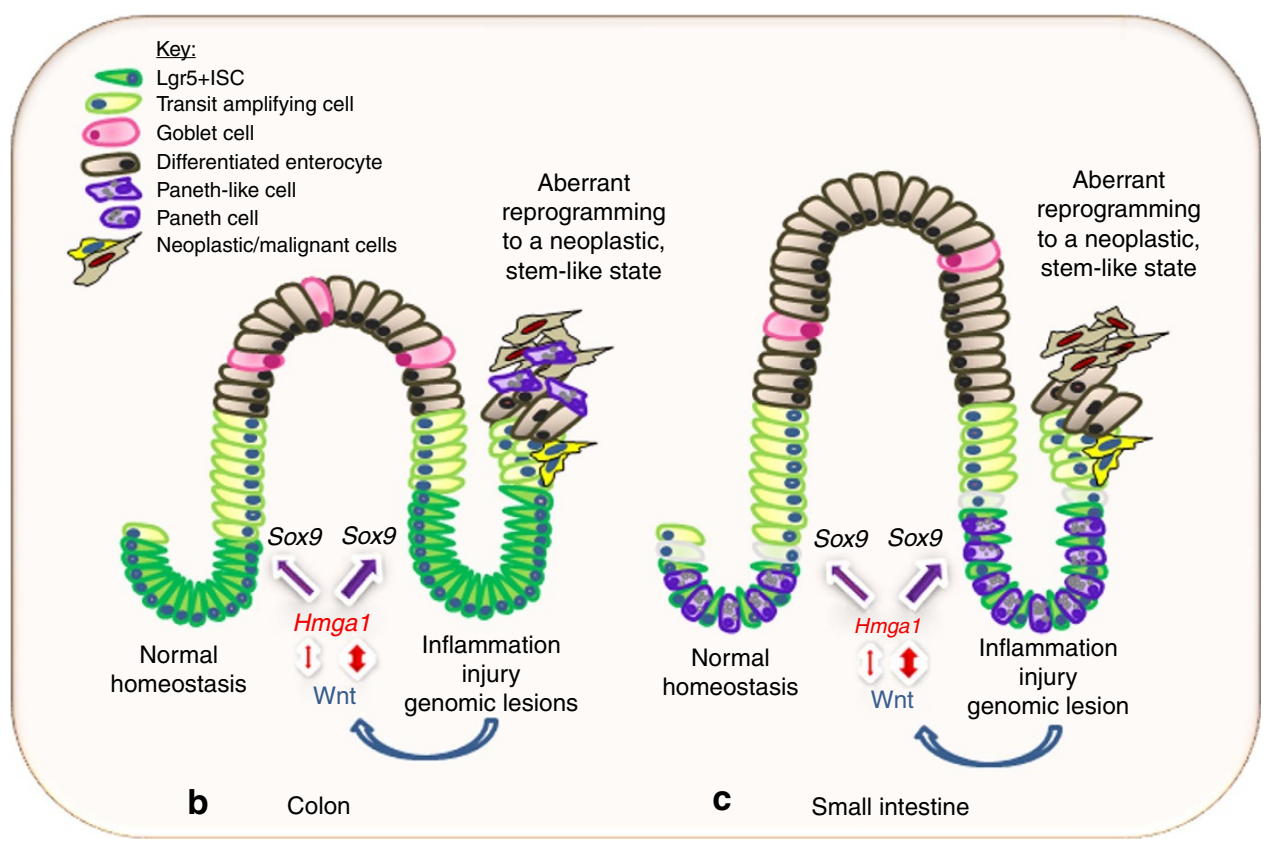

Figure 8 | Hmga1 in normal intestinal homeostasis and reprogramming to neoplasia. (a) There is a significant positive correlation $(r=0.51 ; P=0.008)$ between HMGA1 and SOX9 in control, non-malignant large intestinal epithelium by Spearman's correlation $(n=26)$. There is also a highly significant upregulation of both HMGA1 and SOX9 in colorectal cancer $(n=293, P<0.000001)$. Boxplots, scatterplots and Spearman rank-based correlations were calculated using R statistical software. (b) Model depicting normal ISC function and tissue homeostasis in the large intestine under conditions of tightly regulated Hmgal expression and Wnt signalling (left). Intestinal epithelium homeostasis is disrupted (right) in the setting of aberrant $\mathrm{Hmga1}$ expression, leading to expansion in the ISC compartment, excessive Wnt signalling, and abnormal proliferation, culminating in epithelial reprogramming to neoplastic, transformed cells. (c) Model depicting normal ISC function and tissue homeostasis in the small intestine under conditions of tightly regulated Hmgal expression, Paneth cell differentiation and Wnt signalling (left). Expansion in the ISCs and Paneth cell niche occurs when homeostasis is disrupted (right) in the setting of aberrant Hmga1 expression. This contributes to amplified Wnt signalling, abnormal proliferation, epithelial reprogramming and neoplastic transformation.

are located (Supplementary Fig. 10). Because HMGA1 is overexpressed in diverse epithelial cancers and correlates with cancer stem cell properties in experimental models, we also sought to determine whether HMGA1 and SOX9 are co-regulated in colorectal cancer. Strikingly, both HMGA1 and SOX9 are markedly upregulated in human colorectal cancer $(P<0.0000001)$, although their expression was not correlated in this setting (Fig. 8a). Interestingly, the relative expression and amplitude of HMGA1 mRNA levels are greater than that of SOX9 (Fig. 8a). These data support a model whereby HMGA1 and SOX9 are crucial for normal ISC function, and both become upregulated in carcinogenesis (Fig. 8b,c). These findings also suggest that upregulation of both HMGA1 and SOX9 beyond a threshold may be necessary for early reprogramming of an epithelial cell to a neoplastic cell, while further increases in HMGA1 could drive tumour progression.

\section{Discussion}

HMGA1 expression has been identified among genes most enriched in embryonic and adult stem cells, although its function in these settings had been poorly understood. Our studies reveal a role for Hmgal in both stem cell self-renewal and establishment of a stem cell niche within small intestinal crypts. The HMGA gene family includes HMGA1 (on chromosome 6p21) and HMGA2 (on chromosome 12q15), both of which are highly expressed during embryonic development, but with low or undetectable levels in differentiated tissues ${ }^{13,16,25,26}$. Aberrant expression of HMGA1 occurs in most poorly differentiated human cancers, including gastrointestinal cancers such as colon, gastric, pancreatic and esophageal cancers, and high levels correlate with poor outcomes in diverse tumours ${ }^{13,30-35}$. Notably, a prior study in colorectal cancer identified HMGA1 among the genes most enriched in cancer relative to adjacent, non-malignant tissue ${ }^{33}$. HMGA1 is also required for properties attributed to cancer stem cells, including tumour initiator cells, growth as $3 \mathrm{D}$ spheres and metastatic progression ${ }^{14,18}$. In contrast, HMGA2 overexpression occurs primarily in benign tumours of mesenchymal origin $^{31}$, as well as a subset of malignant tumours $^{52-55}$. The dual role for Hmgal in normal development and poorly differentiated cancers suggests that it regulates cell fate 
decisions, although a detailed understanding of molecular mechanisms involved in these processes was previously unknown.

Here we show that Hmgal amplifies Wnt signalling to drive self-renewal and ISC expansion. Hmgal not only upregulates Wnt agonist receptor genes, but also enhances expression of genes downstream of $\mathrm{WntTcf} 4 / \beta$-catenin. Our results, together with the prior finding that Tcf4 binds to the HMGA1 promoter in colorectal cancer cells ${ }^{56}$, suggest that Hmgal is involved in a 'feed-forward' loop, whereby Tcf4/ $\beta$-catenin induces Hmgal, leading to enhanced Wnt signalling. The expansion of ISCs in our transgenic mouse model was recapitulated in vitro in organoid cultures, which depend on Hmgal for organization into $3 \mathrm{D}$ structures and bud formation. Our transgenic mouse and organoid models provide valuable tools to further dissect downstream pathways regulated by Hmgal in ISCs. In a murine model of gastric cancer, Wnt signalling also upregulates Hmgal (ref. 57). We are the first to show that Hmgal enhances Wnt/ $\beta$-catenin signalling at multiple levels in the pathway, consistent with a feed-forward amplification loop, whereby Wnt induces Hmgal, which in turn, upregulates $\mathrm{Wnt} / \beta$-catenin signalling (Fig. 7).

Our work also uncovered a role for Hmgal in Paneth cell differentiation, mediated in part, through Sox9. Paneth cells constitute an epithelial niche, providing Wnt3a and other signals to maintain ISCs and permit self-renewal within the crypts. Cues from the epithelial and stromal niche are likely to help govern whether Hmgal induces Sox 9 to drive Paneth cell differentiation or other Wnt signals to drive self-renewal. Because adult stem cells can divide asymmetrically, Hmgal could promote ISC division to generate both an identical daughter cell and a differentiated Paneth cell, or even a more fully differentiated transit-amplifying cell. Recent studies indicate that R-spondin 1, another Wnt receptor agonist secreted by intestinal stromal cells, is required for ISC maintenance in mice ${ }^{39,40}$. It remains to be seen whether Hmgal also regulates R-spondin 1 in stromal niche cells. Of note, mesenchymal stem cells within the bone marrow niche also express high levels of HMGA1, although the functional significance of HMGA1 in this setting is not yet known ${ }^{58}$. Prior studies indicate that quiescent, label-retaining cells that are responsible for intestinal epithelial regeneration following injury also differentiate into secretory progenitors and Paneth cells ${ }^{59}$. Hmgal may also function in this setting, although lineage-tracing experiments will be needed to test this.

In addition to establishing a role for Hmgal in maintaining ISC and niche compartments, we also found a positive correlation between HMGA1 and SOX9 in normal human large intestinal epithelium. Moreover, both genes are upregulated in colorectal cancer, indicating that this pathway may contribute to human intestinal carcinogenesis ${ }^{14,33}$. A recent study in the Adenomatous Polyposis Coli $(A p c)^{+1-}$ murine model of intestinal carcinogenesis showed that Hmgal is downstream of the miR-26 tumour suppressor ${ }^{60}$, suggesting that $A P C$ mutations in colorectal cancer lead to HMGA1 induction through loss of miR-26. HMGA1 is also upregulated in the setting of inflammation ${ }^{13}$, and intestinal carcinogenesis is frequently preceded by chronic inflammation and injury ${ }^{61}$. Thus, both inflammatory lesions and genetic alterations ( $A P C$ inactivating mutations) could cooperate to induce $H M G A 1$ during carcinogenesis. Paneth cell expansion also occurs in mice with deletion in the $A p c$ tumour suppressor ${ }^{62}$, and Hmgal could contribute to this phenotype. Although there are no discrete populations of Paneth cells outside of the proximal colon in humans, lysozyme-expressing Paneth-like cells are found in human adenomas ${ }^{62}$. Based on our findings, it is plausible that HMGA1 induces formation of Paneth-like niche cells during colon carcinogenesis in the intestinal epithelium. Studies in murine pancreatic cancer show that SOX9 reprograms acinar cells to ductal cells during carcinogenesis ${ }^{63}$. Similarly, HMGA1 could induce SOX9 and enforce Wnt signalling to drive stem cell properties and reprogram intestinal epithelial cells during carcinogenesis. Although HMGA1 and SOX9 are positively correlated in normal colonic epithelium and both become upregulated in cancer, it is not surprising that the correlation is lost in colorectal cancer. This is consistent with the large body of evidence demonstrating that colon cancer is initiated by the progressive acquisition of mutations in the APC pathway, which include genes upstream of SOX9 and the SOX9 gene itself ${ }^{10,11}$. Thus, there are multiple distinct mechanisms that could give rise to overexpression of HMGA1 and SOX9 in colorectal cancer, and SOX9 may depend, at least in part, on genetic lesions distinct from $H M G A 1$ overexpression in this setting. Together, our work not only reveals a novel function for Hmgal in intestinal homeostasis through self-renewal of ISCs and Paneth cell differentiation, but also sheds light on possible mechanisms involved in Hmgal-mediated neoplastic transformation and intestinal carcinogenesis.

\section{Methods}

Mouse models. The Hmgal transgenic construct and mice have been previously described $^{35,36}$. Female Lgr5-eGFP-IRES-CreERT2 mice ${ }^{6}$ (Jackson Labs) were crossed with male Hmgala transgenics ${ }^{35}$. All mice were on the C57Bl6 background. Animal experiments were conducted in accordance with our institutional Animal Care and Use Committee (protocol\# MO14M187). All mice were housed in a sterile environment where they had free access to food and water as outlined in our institutional guidelines. The ages of the mice were 2-4 months; $\sim 50 \%$ were male. Experiments comparing wild-type to transgenic mice were matched for age and gender in all cases.

Crypt isolation. Crypts were isolated using an established protocol ${ }^{4-7,41}$. First, intestines were flushed with phosphate-buffered saline (PBS) and incised longitudinally after which villi were removed mechanically by scraping. Sections $(1 \mathrm{~cm})$ were incubated in EDTA $(5 \mathrm{mM}) / \mathrm{PBS}$ for $15 \mathrm{~min}$ at $4^{\circ} \mathrm{C}$ per fraction of epithelium. After incubation, the epithelium was separated by vigorous shaking and the remaining intestinal tissue was placed in a new tube for collection of subsequent fractions. After isolation, crypt cells were pelleted, passed through a $70 \mu \mathrm{m}$ cell strainer (unless indicated otherwise), evaluated for purity

microscopically and counted. For purification of single Lgr5-GFP + ISCs, isolated crypts were incubated in culture medium for $45 \mathrm{~min}$ at $37^{\circ} \mathrm{C}$, followed by trituration with a glass pipette. Dissociated cells were passed through a cell strainer with a pore size of $20 \mu \mathrm{m}$. GFP + and GFP - cells were sorted by FACS Aria model (BD Biosciences).

Organoid culture and re-plating assay. Mouse organoids were established and maintained from isolated crypts of the proximal small intestine or from Lgr5 + ISCs isolated as single cells ${ }^{4-8,41}$. The basic culture medium (advanced Dulbecco's modified Eagle's medium/F12 supplemented with penicillin/streptomycin, $10 \mathrm{mmoll}^{-1}$ HEPES, 13 Glutamax, 13 B27 (all from Life Technologies), and $1 \mathrm{mmoll}^{-1} \mathrm{~N}$-acetylcysteine (Sigma)) was supplemented with $50 \mathrm{ng} \mathrm{ml}^{-1}$ murine recombinant EGF (Peprotech), R-spondin $1\left(1 \mu \mathrm{g} \mathrm{ml}^{-1}\right)$ and Noggin

$\left(10 \mathrm{ng} \mathrm{ml}^{-1}\right)$. Wnt inhibitors C59 and IWP-2 are commercially available (Abcam) Conditioned media was produced using HEK 293 T cells stably transfected with HA-mouse Rspo1-Fc (a gift from Calvin Kuo, Stanford University). Advanced DMEM/F12 media supplemented with penicillin/streptomycin, $10 \mathrm{mmoll}^{-1}$ HEPES, and 13 Glutamax was conditioned for 1 week. For the re-plating assay, an equal number of cells from each model were isolated by mechanical dispersion with a $10 \mathrm{ml}$ pipet after 3-5 days in culture. Cells were then counted and re-plated using the conditions described above.

Lentivirus and transduction. The FUGW control vector (Addgene plasmid \# 14883) and FUGW-Hmgal lentiviral vectors ${ }^{36,64}$ have been described ${ }^{14,16,18}$. The shRNA interference plasmid for Hmgal (\#TRCN0000182169; the RNAi Consortium/TRC) has been described ${ }^{14,16,18}$. The empty shRNA vector was used as a control. For inducible silencing, pTRIPz-Hmgal-shRNA linked to RFP reporter was engineered to be inducible by tetracycline or analogues (Tet-On) and produces tightly regulated induction of shRNA expression in the presence of doxycycline $\left(0.5 \mu \mathrm{g} \mathrm{ml}^{-1}\right)$. The annealed Hmgal-shRNA oligonucleotides were cloned into linearized pTRIPZ empty vector (Open Biosystems catalogue number RHS4750). For pTRIPz-Hmgal-shRNA transductions, puromycin $\left(2 \mu \mathrm{g} \mathrm{ml}^{-1}\right)$ was added to media after 2-3 days for selection. For all lentiviral transductions, we modified an established protocol ${ }^{65}$ using magnetic nanoparticles (ViroMag R/L, OZ Bioscience, Inc.) and a magnetic plate (ViroMag R/L, OZ Bioscience, Inc., catalogue number: 
MF10000) to transduce crypt cells and organoids. Organoid fragments were seeded with $150 \mu$ l transduction medium into 48 -well plates. Virus was added with viroMag $\mathrm{R} / \mathrm{L}$ solution for $15 \mathrm{~min}$ at room temperature $(2,500-3,000$ virus particles per cells) to cells. The cell culture plate was placed on the magnetic plate for $30-60 \mathrm{~min}$ in a $37^{\circ} \mathrm{C}$ tissue culture incubator. Cells were then incubated overnight at $37^{\circ} \mathrm{C}$. Organoid fragments and transduction media were then transferred to a $1.5 \mathrm{ml}$ tube for centrifugation at $900 \mathrm{~g}$ for $5 \mathrm{~min}$. The supernatant was discarded and tubes containing the pellet was placed on ice for $5 \mathrm{~min}$. Next, $120 \mu \mathrm{l}$ of matrigel was added and the pellet was resuspended by pipetting slowly up and down. Drops (30 $\mu \mathrm{l})$ of basement matrix-cell mixtures were seeded into a new 48-well plate and incubated at $37^{\circ} \mathrm{C}$ for $5-15 \mathrm{~min}$ until the basement matrix solidified. Media (ENRWntNic) ${ }^{6}$ was then added to each well and placed into a tissue culture incubator. Common ENR media ${ }^{6}$ was used and changed every 2-3 days beginning 4-6 days after the transduction.

Gene expression analysis and chromatin immunoprecipitation. Wnt signalling molecule gene expression was detected by qPCR $^{14-16}$ (see Supplementary Table 1 for primer sequence). Reactions were performed using the SYBR Green PCR Master Mix (Applied Biosystems) with the ABI 7500 qRT-PCR machine. Gapdh mRNA was assessed as the loading control ${ }^{14}$. For ChIP, primers were designed using the sequence data from MatInspector in silico transcription factor-binding site prediction algorithm ${ }^{66}$ with amplicon sizes of around $200 \mathrm{bp}$. Both positive control antibodies (histone $\mathrm{H} 3$ ) and negative control antibodies (IgG) as well as a negative control promoter sequence (from the Hprt gene) were included ${ }^{36,67}$. (See Supplementary Table 2 for ChIP primer sequences and antibodies.) All experiments were done in triplicate and performed at least twice.

Western blots. Total protein from organoid cultures or tissues was isolated using RIPA buffer (Sigma, USA). Lysates were centrifuged and separated on a gradient gel (8-12\%) via SDS-polyacrylamide gel electrophoresis, blotted onto a membrane (polyvinylidene fluoride; Bio Rad, USA), and analysed using specific antibodies (Supplementary Table 3). Bands were visualized using enhanced chemiluminescence (ECL Kit, Amersham Biosciences, USA). Images of all uncropped western blots can be found in Supplementary Fig. 11.

\section{Statistical analysis. Data from all experiments were assessed for normal} distribution (Ryan-Joyner and D'Agostino-Pearson tests), and when normally distributed, compared using the two-tailed, Student's $t$-test. The Mann-Whitney test was used for data that were not normally distributed. $P<0.05$ was considered significant. Experiments that were technical failures, such as in vitro cultures in which controls did not grow, were excluded from the statistical analysis. Sample sizes were chosen empirically based on previous experiments; no statistical methods were used to predetermine sample sizes. Investigators were not blinded. Mice were analysed based on genotype; randomization was not performed.

TCGA gene expression analysis. Transcript abundance for HMGA1 and SOX9 from human RNA-Seq data sets obtained from 293 primary colorectal cancers and 26 non-malignant colonic epithelial samples were assessed using RNA-Seq expectation maximization (RSEM) normalization. IluminaGa RNA-Seq data are available through TCGA data portal (Archive: COADREAD.rnaseqv2_illuminaga_ rnaseqv2_unc_edu_Level_3_RSEM_genes_normalized_data.data.txt).

Expression levels were converted to $\log$ (base 2 ) after adding a small quantity (0.001) to account for zeros in the data. Boxplots, scatterplots and Spearman rank-based correlations were calculated using R statistical software suite using standard functions ${ }^{67}$.

Immunohistochemistry. Haematoxylin \& eosin and IHC staining of organoid and intestinal sections were performed after fixing organoid cultures in formalin at $4{ }^{\circ} \mathrm{C}$ before paraffin or frozen embedding ${ }^{14,16,68,69}$. (See Supplementary Table 3 for list of primary antibodies.)

Time-lapsed confocal imaging and image analysis. Crypt cells were isolated from WT and Hmgal mice separately, resuspended in matrigel, and cultured on glass-bottom 24 -well plates ${ }^{41}$. Crypt cells were fixed either immediately (day 0 ) after seeding or following culture for 6 days $^{42-45}$. Green fluorescence of Lgr5 + $(\mathrm{GFP}+)$ cells was amplified by labelling anti-GFP antibody (00-106-215, Rockland Immunochemicals Inc. Limerick, PA) followed by staining with Alexa Fluor 488 anti-Goat secondary antibody (A-11055, ThermoFisher Scientific, Waltham, MA). Entire organoids were visualized with Alexa Fluor 568 Phalloidin (A12380, ThermoFisher Scientific) and Hoescht 33258 labelling (H3569, ThermoFisher Scientific) using confocal imaging ${ }^{42-45}$. Images were collected using a Solamere Technology Group spinning disk confocal microscope with a $\times 10$ objective (Zeiss Microimaging). A combination of $\mu$ Manager and Piper (Stanford Photonics) imaging software was used for imaging acquisition ${ }^{42-45}$. All organoids were imaged using identical excitation and detection settings per fluorescence channel. ImageJ was used for post-acquisition image analysis and quantification of Lgr5 + (green channel) Z-projected surface areas $\left(\mu \mathrm{m}^{2}\right)$ per organoid. Briefly, $2 \mu \mathrm{m}$-step Z-stacks of single organoids were contrasted to exclude background fluorescence, maximaprojected to create a single plane image per channel, after which a threshold was applied for specific signal within organoid boundaries. Projected monochromatic surface area was then measured within each imaged organoid, and expansion rate of Lgr5 + cell population in WT and Hmgal mice, separately, was calculated by normalization of day 6 projected area over day 0 projected area in the green channel.

Cell culture. Caco-2 and HEK 293 cell lines (from ATCC) were cultured in highglucose DMEM, supplemented with $4 \mathrm{mM}$ glutamine, $50 \mathrm{U} \mathrm{ml}^{-1}$ penicillin, $50 \mu \mathrm{g} \mathrm{ml}^{-1}$ streptomycin (all from Invitrogen) and 10\% fetal bovine serum. All cell lines were routinely tested for mycoplasma contamination with the MycoAlert Mycoplasma Detection Kit (Lonza). For cell line authentication, short-tandem repeat analysis was performed on isolated genomic DNA with the GenePrint 10 System from Promega, and peaks were analysed using GeneMarker HID from Softgenetics. Allele calls were searched against short-tandem repeat databases maintained by ATCC (www.atcc.org) and DSMZ (www.dsmz.de).

Data availability. The TCGA RNA-seq data (Fig. 8a) have been deposited in EZID (at the California Digital Library) with identifier: Broad GDAC Firehose stddata_2013_08_09 run, doi:10.7908/C17W6BB3. Relevant primary data are presented in the manuscript and supplementary files; all primary data are available from the authors on request.

\section{References}

1. Clevers, H. The intestinal crypt, a prototype stem cell compartment. Cell 154, 274-284 (2013).

2. Koo, B. K. et al. Controlled gene expression in primary Lgr5 organoid cultures. Nat. Methods 9, 81-83 (2011).

3. Koo, B. K., Sasselli, V. \& Clevers, H. Retroviral gene expression control in primary organoid cultures. Curr. Protoc. Stem Cell Biol. 27, 5A.6.1-5A.6.8 (2013).

4. Sato, T. \& Clevers, H. Growing self-organizing mini-guts from a single intestinal stem cell: mechanism and applications. Science 340, 1190-1194 (2013).

5. Koo, B. K. \& Clevers, H. Stem cells marked by the R-spondin receptor LGR5. Gastroenterology 147, 289-302 (2014).

6. Barker, N. et al. Identification of stem cells in small intestine and colon by marker gene Lgr5. Nature 449, 1003-1007 (2007).

7. Schuijers, J. et al. Ascl2 acts as an R-spondin/Wnt-responsive switch to control stemness in intestinal crypts. Cell Stem Cell 16, 158-170 (2015).

8. Cheung, E. C. et al. TIGAR is required for efficient intestinal regeneration and tumorigenesis. Dev. Cell 25, 463-477 (2013).

9. Farin, H. F. et al. Visualization of a short-range Wnt gradient in the intestinal stem-cell niche. Nature 530, 340-343 (2016).

10. Fearon, E. R. Molecular genetics of colorectal cancer. Annu. Rev. Pathol. 6 , 479-507 (2011)

11. Vogelstein, B. et al. Cancer genome landscapes. Science 339, 1546-1558 (2013)

12. Reeves, R., Edberg, D. D. \& Li, Y. Architectural transcription factor HMGI(Y) promotes tumor progression and mesenchymal transition of human epithelial cells. Mol. Cell Biol. 21, 575-594 (2001).

13. Resar, L. M. The high-mobility group A1 gene: transforming inflammatory signals into cancer? Cancer Res. 70, 436-439 (2010).

14. Belton, A. et al. HMGA1 induces intestinal polyposis in transgenic mice and drives tumor progression and stem cell properties in colon cancer cells. PLoS ONE 7, e30034 (2012).

15. Schuldenfrei, A. et al. HMGA1 drives stem cell, inflammatory pathway, and cell cycle progression genes during lymphoid tumorigenesis. BMC Genomics 12, 549 (2011).

16. Shah, S. N. et al. HMGA1 reprograms somatic cells into pluripotent stem cells by inducing stem cell transcriptional networks. PLoS ONE 7, e48533 (2012).

17. Shah, S. N. \& Resar, L. M. High mobility group A1 and cancer: potential biomarker and therapeutic target. Histol. Histopathol. 27, 567-579 (2012)

18. Shah, S. N. et al. HMGA1: a master regulator of tumor progression in triplenegative breast cancer cells. PLoS ONE 8, e63419 (2013).

19. Johnson, K. R., Lehn, D. A., Elton, T. S., Barr, P. J. \& Reeves, R. Complete murine cDNA sequence, genomic structure, and tissue expression of the high mobility group protein HMG-I(Y). J. Biol. Chem. 263, 18338-18342 (1988).

20. Friedmann, M., Holth, L. T., Zoghbi, H. Y. \& Reeves, R. Organization, inducible-expression and chromosome localization of the human HMG-I(Y) nonhistone protein gene. Nucleic Acids Res. 21, 4259-4267 (1993).

21. Pedulla, M. L., Treff, N. R., Resar, L. M. \& Reeves, R. Sequence and analysis of the murine Hmgiy (Hmga1) gene locus. Gene 271, 51-58 (2001).

22. Thanos, D. \& Maniatis, T. Virus induction of human IFN beta gene expression requires the assembly of an enhanceosome. Cell 83, 1091-1100 (1995).

23. Falvo, J. V., Thanos, D. \& Maniatis, T. Reversal of intrinsic DNA bends in the IFN beta gene enhancer by transcription factors and the architectural protein HMG I(Y). Cell 83, 1101-1111 (1995). 
24. Huth, J. R. et al. The solution structure of an HMG-I(Y)-DNA complex defines a new architectural minor groove binding motif. Nat. Struct. Biol. 4, 657-665 (1997).

25. Chiappetta, G. et al. High level expression of the HMGI (Y) gene during embryonic development. Oncogene 13, 2439-2446 (1996).

26. Ben-Porath, I. et al. An embryonic stem cell-like gene expression signature in poorly differentiated aggressive human tumors. Nat. Genet. 40, 499-507 (2008).

27. Zhou, G. et al. The pattern of gene expression in human CD $34^{+}$stem/ progenitor cells. Proc. Natl Acad. Sci. USA 98, 13966-13971 (2001).

28. Chou, B. K. et al. Efficient human iPS cell derivation by a non-integrating plasmid from blood cells with unique epigenetic and gene expression signatures. Cell Res. 21, 518-529 (2011).

29. Munoz, J. et al. The Lgr5 intestinal stem cell signature: robust expression of proposed quiescent ' +4 ' cell markers. EMBO J. 31, 3079-3091 (2012).

30. Wood, L. J. et al. $H M G-I / Y$, a new c-Myc target gene and potential oncogene. Mol. Cell Biol. 20, 5490-5502 (2000).

31. Fusco, A. \& Fedele, M. Roles of HMGA proteins in cancer. Nat. Rev. Cancer 7, 899-910 (2007).

32. Roy, S. et al. HMGA1 overexpression correlates with relapse in childhood B-lineage acute lymphoblastic leukemia. Leuk. Lymphoma 54, 2565-2567 (2013).

33. Grade, M. et al. Gene expression profiling reveals a massive, aneuploidydependent transcriptional deregulation and distinct differences between lymph node-negative and lymph node-positive colon carcinomas. Cancer Res. 67, 41-56 (2007).

34. Hristov, A. C. et al. HMGA1 correlates with advanced tumor grade and decreased survival in pancreatic ductal adenocarcinoma. Mod. Pathol. 23, 98-104 (2010).

35. Xu, Y. et al. The HMG-I oncogene causes highly penetrant, aggressive lymphoid malignancy in transgenic mice and is overexpressed in human leukemia. Cancer Res. 64, 3371-3375 (2004).

36. Tesfaye, A. et al. The high-mobility group A1 gene up-regulates cyclooxygenase 2 expression in uterine tumorigenesis. Cancer Res. 67, 3998-4004 (2007).

37. Dominici, M. et al. Transgenic mice with pancellular enhanced green fluorescent protein expression in primitive hematopoietic cells and all blood cell progeny. Genesis 42, 17-22 (2005).

38. Clevers, H. \& Nusse, R. Wnt/beta-catenin signaling and disease. Cell 149, 1192-1205 (2012).

39. Kabiri, Z. et al. Stroma provides an intestinal stem cell niche in the absence of epithelial Wnts. Development 141, 2206-2215 (2014).

40. Wu, C. et al. RSPO2-LGR5 signaling has tumour-suppressive activity in colorectal cancer. Nat. Commun. 5, 3149 (2014).

41. Sato, T. \& Clevers, H. Primary mouse small intestinal epithelial cell cultures. Methods Mol. Biol. 945, 319-328 (2013).

42. Nguyen-Ngoc, K. V. et al. 3D culture assays of murine mammary branching morphogenesis and epithelial invasion. Methods Mol. Biol. 1189, 135-162 (2015).

43. Huebner, R. J., Lechler, T. \& Ewald, A. J. Developmental stratification of the mammary epithelium occurs through symmetry-breaking vertical divisions of apically positioned luminal cells. Development 141, 1085-1094 (2014).

44. Ewald, A. J. Practical considerations for long-term time-lapse imaging of epithelial morphogenesis in three-dimensional organotypic cultures. Cold Spring Harb. Protoc. 2013, 100-117 (2013).

45. Cheung, K. J. et al. Polyclonal breast cancer metastases arise from collective dissemination of keratin 14-expressing tumor cell clusters. Proc. Natl Acad. Sci. USA 113, E854-E863 (2016).

46. Fuerer, C. \& Nusse, R. Lentiviral vectors to probe and manipulate the Wnt signaling pathway. PLoS ONE 5, e9370 (2010).

47. Mori-Akiyama, Y. et al. SOX9 is required for the differentiation of paneth cells in the intestinal epithelium. Gastroenterology 133, 539-546 (2007).

48. Formeister, E. J. et al. Distinct SOX9 levels differentially mark stem/progenitor populations and enteroendocrine cells of the small intestine epithelium. Am. J. Physiol. Gastrointest. Liver Physiol. 296, G1108-G1118 (2009).

49. Bastide, P. et al. Sox9 regulates cell proliferation and is required for Paneth cell differentiation in the intestinal epithelium. J. Cell. Biol. 178, 635-648 (2007).

50. Roche, K. C. et al. SOX 9 maintains reserve stem cells and preserves radioresistance in mouse small intestine. Gastroenterology 149, 1553-1563.e10 (2015).

51. Gracz, A. D. et al. A high-throughput platform for stem cell niche co-cultures and downstream gene expression analysis. Nat. Cell. Biol. 17, 340-349 (2015).

52. Di Cello, F. et al. HMGA2 participates in transformation in human lung cancer. Mol Cancer Res. 6, 743-750 (2008).

53. Hristov, A. C. et al. HMGA2 protein expression correlates with lymph node metastasis and increased tumor grade in pancreatic ductal adenocarcinoma. Mod. Pathol. 22, 43-49 (2009).

54. Morishita, A. et al. HMGA2 is a driver of tumor metastasis. Cancer Res. 73, 4289-4299 (2013).

55. Kugel, S. et al. SIRT6 suppresses pancreatic cancer through control of Lin28b. Cell 165, 1401-1415 (2016).

56. Bush, B. M., Brock, A. T., Deng, J. A., Nelson, R. A. \& Sumter, T. F. The Wnt/beta-catenin/TCF-4 pathway upregulates $H M G A 1$ expression in colon cancer. Cell Biochem. Funct. 31, 228-236 (2013).
57. Akaboshi, S. et al. HMGAl is induced by Wnt/beta-catenin pathway and maintains cell proliferation in gastric cancer. Am. J. Pathol. 175, 1675-1685 (2009).

58. Ullah, M. et al. Transdifferentiation of mesenchymal stem cells-derived adipogenic-differentiated cells into osteogenic- or chondrogenic-differentiated cells proceeds via dedifferentiation and have a correlation with cell cycle arresting and driving genes. Differentiation 85, 78-90 (2013).

59. Buczacki, S. J. et al. Intestinal label-retaining cells are secretory precursors expressing Lgr5. Nature 495, 65-69 (2013).

60. Zeitels, L. R. et al. Tumor suppression by miR-26 overrides potential oncogenic activity in intestinal tumorigenesis. Genes Dev. 28, 2585-2590 (2014).

61. Wang, D. \& DuBois, R. N. The role of anti-inflammatory drugs in colorectal cancer. Annu. Rev. Med 64, 131-144 (2013).

62. Feng, Y. et al. Sox9 induction, ectopic Paneth cells, and mitotic spindle axis defects in mouse colon adenomatous epithelium arising from conditional biallelic Apc inactivation. Am. J. Pathol. 183, 493-503 (2013).

63. Kopp, J. L. et al. Identification of Sox9-dependent acinar-to-ductal reprogramming as the principal mechanism for initiation of pancreatic ductal adenocarcinoma. Cancer Cell 22, 737-750 (2012).

64. Lois, C., Hong, E. J., Pease, S., Brown, E. J. \& Baltimore, D. Germline transmission and tissue-specific expression of transgenes delivered by lentiviral vectors. Science 295, 868-872 (2002).

65. Andersson-Rolf, A., Fink, J., Mustata, R. C. \& Koo, B. K. A video protocol of retroviral infection in primary intestinal organoid culture. J. Vis. Exp. 2014, e51765 (2014).

66. Cartharius, K. et al. MatInspector and beyond: promoter analysis based on transcription factor binding sites. Bioinformatics 21, 2933-2942 (2005).

67. Hillion, J. et al. The high mobility group A1 (HMGA1) gene is highly overexpressed in human uterine serous carcinomas and carcinosarcomas and drives matrix metalloproteinase-2 (MMP-2) in a subset of tumors. Gynecol. Oncol. 141, 580-587 (2016).

68. Hillion, J. et al. The high-mobility group A1a/signal transducer and activator of transcription-3 axis: an achilles heel for hematopoietic malignancies? Cancer Res. 68, 10121-10127 (2008)

69. van Es, J. H., de Geest, N., van de Born, M., Clevers, H. \& Hassan, B. A. Intestinal stem cells lacking the Math1 tumour suppressor are refractory to Notch inhibitors. Nat. Commun. 1, 18 (2010).

\section{Acknowledgements}

We dedicate this work to the memory of our esteemed colleague and co-author, Dr David Huso. His generosity, kindness and intellect were immense and will continue to inspire all who knew him. This work was supported by grants from the National Institute of Health (CA2149550, CA164677, DK104344) and Maryland Stem Cell Research Fund (2011-MSCRFE-0102, 2015-MSCRFE-1759)

\section{Author contributions}

L.X. designed and performed most of the experiments, analysed the data and prepared the manuscript; T.H., A.B., Y.-T.C., Q.G., X.Z., W.K. and D.L.H. performed experiments and analysed the data, L.C. analysed TCGA databases, S.S. and A.F. helped with organoid cultures; D.G. and A.J.E. designed and performed experiments with confocal, single cell and organoid imaging, and analysed confocal microscopic data, L.M.S.R. conceived the study, supervised the project, edited and finalized the manuscript.

\section{Additional information}

Supplementary Information accompanies this paper at http://www.nature.com/ naturecommunications

\section{Competing interests: The authors declare no competing financial interests.}

Reprints and permission information is available online at http://npg.nature.com/ reprintsandpermissions/

How to cite this article: Xian, L. et al. HMGAl amplifies Wnt signalling and expands the intestinal stem cell compartment and Paneth cell niche. Nat. Commun. 8, 15008 doi: 10.1038/ncomms15008 (2017).

Publisher's note: Springer Nature remains neutral with regard to jurisdictional claims in published maps and institutional affiliations.

This work is licensed under a Creative Commons Attribution 4.0 International License. The images or other third party material in this article are included in the article's Creative Commons license, unless indicated otherwise in the credit line; if the material is not included under the Creative Commons license, users will need to obtain permission from the license holder to reproduce the material. To view a copy of this license, visit http://creativecommons.org/licenses/by/4.0/ 